\title{
The Transition from a Drèze Equilibrium to a Walrasian Equilibrium *
}

\author{
P. Jean-Jacques Herings ${ }^{\dagger}$ \\ Gerard van der Laan ${ }^{\ddagger}$ \\ Richard Venniker $§$
}

\begin{abstract}
In this paper a continuous time price and quantity adjustment process is considered for an economy facing price rigidities. In the short run prices are assumed to be completely fixed and the markets are cleared by quantity adjustments until a fixed price equilibrium is reached where every market is typically characterized by either supply rationing or demand rationing. Next prices are assumed to move upwards in case of demand rationing on a market and downwards when supply rationing occurs. Markets are kept in equilibrium by infinitesimal quantity adjustments such that at every moment in time a fixed price equilibrium results. Using only standard assumptions on the primitive concepts of the economy it is shown that the price and quantity adjustment process indeed converges to a fixed price equilibrium for the initially given prices. Moreover, in the long run, when prices are allowed to change, the process is shown to reach a Walrasian equilibrium. A simplicial algorithm is developed to approximate the price and quantity adjustment process arbitrarily close. It is shown that the path of price systems and rationing schemes generated by the algorithm converges to the path of the adjustment process.
\end{abstract}

${ }^{*}$ The authors would like to thank Jacques H. Drèze for his valuable comments on a previous draft of this paper. This research is part of the Research Program "Competition and Cooperation". This research has been partially supported by a grant "Actions de Recherche Concertées" $n^{\circ}$ 93/98-162 of the Ministry of Scientific Research of the Belgian French Speaking Community.

${ }^{\dagger}$ P.J.J. Herings, Department of Economics and IRES, Université Catholique de Louvain, Belgium.

${ }^{\ddagger} \mathrm{G}$. van der Laan, Department of Econometrics and Tinbergen Institute, Free University, De Boelelaan 1105, 1081 HV Amsterdam, The Netherlands

${ }^{\S}$ R.J.G. Venniker, Department of Econometrics and Tinbergen Institute, Free University, De Boelelaan 1105, 1081 HV Amsterdam, The Netherlands. This author is financially supported by the Netherlands Organization for Scientific Research (NWO) 


\section{Introduction}

Probably the best known process of price adjustment in an exchange economy is the Walrasian tatonnement process. In a situation where demand does not equal supply on the markets of some commodities, the price on any market is adjusted according to the sign of the excess demand. Furthermore, as long as a non-zero excess demand prevails on some market, no exchange of commodities takes place. Eventually, it is hoped, a situation of zero excess demand will be obtained such that trade can take place.

The Walrasian tatonnement process has several shortcomings. One of the drawbacks of the Walrasian tatonnement process is that convergence to a Walrasian equilibrium price system can not be guaranteed in general. In this paper, convergence is always meant in a global sense, so convergence to some equilibrium of the economy, possibly far away from the starting point. Examples of economies for which the Walrasian tatonnement process does not converge have been given by Scarf [24]. It has been shown by Saari and Simon [23] that convergence of a process based on the excess demand function can only be guaranteed when it uses almost all of the information provided by the excess demand function and its first derivatives. A well-known process that satisfies this condition is the global Newton method by Smale [26], which converges to an equilibrium price system when started at the boundary of the price space. The processes by van der Laan and Talman [16], [17] and Kamiya [13] not only use local information about the excess demand, but are also based on information of the starting price system. As a consequence, it can be shown that the process of Kamiya converges under standard assumptions plus a reasonably mild boundary condition on the total excess demand function for a generic starting price system (see Kamiya [13]), while for every starting price system the process of van der Laan and Talman converges for a generic exchange economy satisfying the standard conditions (see Herings [9]). A process which is locally convergent for a generic exchange economy satisfying standard assumptions is given in Mukherji [22].

Even when convergence takes place, it will take some time before the equilibrium price system has been reached. Until then, demand is not equal to supply on every market and trade is excluded by the Walrasian tatonnement process, as well as the other processes described above. A related drawback is that the relevant market signals for an adjustment process in an economy are based on the effective demand associated with a Drèze equilibrium (introduced by Drèze (1975)), as has been noticed in Veendorp [28], and not on the notional demand used in the processes above. In a model with three commodities and two consumers and with the total excess demand function satisfying a gross substitutability condition, Veendorp has shown the convergence of an adjustment process that is based on effective excess demands and which follows the path of (unique) Drèze equilibria (see also Laroque [18] for a correction of the convergence proof). Therefore, trade according to the 
prevailing Drèze equilibrium is possible at each point in time. In general, however, such a process does not necessarily converge to a Walrasian equilibrium price system and even chaotic behaviour may be expected as argued in Day and Pianigiani [3] and confirmed in a more complicated model in Böhm [2]. A model closely related to the one of Veendorp is considered in Movshovich [21]. Movshovich considers a process in discrete time, where at each point in time the price of a randomly chosen commodity is adjusted on the basis of the effective excess demand in the previous period at the (unique) Drèze equilibrium at the current price system. Also in the model of Movshovich gross substitutability like conditions are needed in order to guarantee convergence. In Herings, van der Laan, Talman and Venniker [11] an adjustment proces is proposed such that a Drèze equilibrium prevails in the economy at any point generated by the process, and in which prices are adjusted according to the market situation at the Drèze equilibrium. That process is inspired by recent experiences in Eastern European countries and starts from a situation where the price level of the non-numeraire commodities is so low that complete demand rationing is necessary to equilibrate the markets. Next the process generates a path of Drèze equilibria, characterized by the absence of supply rationing, until a Walrasian equilibrium is reached. Contrary to other price adjustment processes based on effective excess demands, this process converges to a Walrasian equilibrium price system under standard assumptions with respect to the economy. Therefore, unlike the other papers, it is for instance not assumed that given some price system there is a unique Drèze equilibrium associated to it.

Also in this paper a price and quantity adjustment process is presented that follows a path of Drèze equilibria. However, now the starting point of the process is a historically given price system and quantity constraints which may not, and will not in general, constitute a Drèze equilibrium. Initially, only the quantity constraints are adjusted, based on the paradigm that quantity adjustments take place much faster than price adjustments. The quantity adjustments are related to the excess demands on the markets of the nonnumeraire commodities and the values of the quantity constraints. This part of the process proceeds until a Drèze equilibrium with respect to the initial fixed price system has been reached, and it coincides with the quantity adjustment process given in Herings [10]. It is referred to as the short-term quantity adjustment process. Notice that this part of the process is of the tatonnement type, since markets are out of equilibrium and no trade is assumed to occur. Unlike the Drèze equilibrium which is the starting point of the process in Herings, van der Laan, Talman, and Venniker [11], the Drèze equilibrium reached is usually not characterized by complete demand rationing and might also have markets with supply rationing. Once a Drèze equilibrium has been obtained, the process proceeds by adjusting the prices. As long as demand (supply) rationing on the market of a commodity prevails, the corresponding price will be increased maximally (minimally) relative to the initial price system, and the rationing scheme is infinitesimally adjusted such that any 
point on the path reached by the process is a Drèze equilibrium given the actual price flexibility. This path of Drèze equilibria either ends with a second Drèze equilibrium with respect to the initial price system or with a Walrasian equilibrium. In the first case, the process proceeds with a short-term quantity adjustment process, until a third Drèze equilibrium with respect to the initial fixed price system has been reached. It will be shown that eventually a Drèze equilibrium at the initial price system will be reached from where the path of Drèze equilibria leads to a Walrasian equilibrium.

It is interesting to compare the above adjustment process to a process in Drèze [7]. Drèze's process starts with historically given prices and quantity constraints on the supply side. He also assumes quantities to move much faster than prices. Drèze shows that after the adjustment of quantities a situation is reached where no market is in excess supply, while positive excess demands may occur on some markets facing no longer any quantity constraints on the supply side. Next, for the markets with positive excess demands the prices will adjust upwards. Contrary, in our model of price and quantity adjustments these markets would be cleared by quantity constraints on the demand side, while price adjustments would be made after all markets have been cleared. Under the assumptions that there are no inferior goods and that the marginal propensity to consume is less than one, Drèze shows convergence of his process to a Drèze equilibrium without demand rationing on any market.

Although the adjustment process of the present paper addresses several shortcomings of existing adjustment processes, it is clear that other problems like the informational requirements needed to make the adjustments or the non-speculative behaviour of economic agents remain. In this respect it is interesting to mention that these informational requirements can be weakend for the Walrasian tatonnement process. In the adjustment process considered in Keisler [14] and Keisler [15] there is decentralization with respect to trades and price adjustments in the sense that the set of trades available to an agent does not depend on the commodity holdings of the other agents in the economy and that at each point in time the prices are adjusted on the basis of the excess demand of a single consumer. Nevertheless, Keisler's process approximates the Walrasian tatonnement process as closely as desired. It seems possible that a similar approach could also weaken the informational requirements for the adjustment process under consideration in this paper.

The convergence proof of the adjustment process described in this paper is based on some techniques of simplicial approximation, as initiated by Scarf [25]. First, an artificial function of excess demands, called the reduced total excess demand function, is constructed. This function is constructed such that the points reached by the adjustment process are zero points of this function. Subsequently, a simplicial algorithm is described and is shown to generate a path of zero points for a piecewise linear approximation of the reduced total excess demand function. This path is then shown to yield an approximation of the desired 
adjustment process. The inaccuracy of the approximation can be made arbitrarily small. A direct analysis of the exact adjustment process is also possible using methods of differential topology. In fact, the convergence of the short-term quantity adjustment process to a Drèze equilibrium has been shown in Herings [10] for a generic $C^{2}$ economy with the economy being parametrized by the initial endowments. The complexity of these proofs made us refrain from a similar analysis for the current process and led us to be content with the $\varepsilon$-result obtained using the technique of simplicial approximation.

The paper has been organized as follows. In Section 2 the model is described and the definition of a Drèze equilibrium is stated. In Section 3 we define the reduced total excess demand function and derive some properties of it that are used in subsequent sections. In Section 4 the adjustment process is discussed and illustrated by a numerical example. In Section 5 some techniques of simplicial approximation are described. Based on these techniques, the convergence of the adjustment process related to a piecewise linear approximation of the reduced total excess demand function is shown. Finally, in Section 6 we show that the inaccuracy with which this path follows the adjustment process as described in Section 4 can be made arbitrarily small. In fact, it is shown that the approximate paths converge to the path of the exact adjustment process if the latter is well-defined.

\section{The model}

For ease of notation, in the sequel we denote the set of indices $\{1, \ldots, k\}$ by $I_{k}$, the set of indices $\{0,1, \ldots, k\}$ by $I_{k}^{0}, \mathbb{R}_{+}^{k}=\left\{x \in \mathbb{R}^{k} \mid x_{j} \geq 0, \forall j \in I_{k}\right\}$ and $\mathbb{R}_{++}^{k}=\left\{x \in \mathbb{R}^{k} \mid x_{j}>0\right.$, $\left.\forall j \in I_{k}\right\}$. We consider an exchange economy $\mathcal{E}=\left(\left\{X^{i}, \succeq^{i}, w^{i}\right\}_{i=1}^{m}, \tilde{p}\right)$. In this economy there are $m$ consumers, indexed $i=1, \ldots, m$, and $n+1$ commodities, indexed $j=1, \ldots, n+1$. Each consumer $i \in I_{m}$ is characterized by a consumption set $X^{i}$, a preference preordering $\succeq^{i}$ on $X^{i}$, and a vector of initial endowments $w^{i}$. The vector $w$ is defined by $w=\sum_{i \in I_{m}} w^{i}$.

In this paper we assume that the economy $\mathcal{E}$ is initially faced with a completely fixed price system, determined by the vector $\tilde{p} \in \mathbb{R}_{++}^{n+1}$. We take one of the commodities, say commodity $n+1$, as the numeraire commodity having a price equal to 1 , so $\tilde{p}_{n+1}=1$. In the short run the prices are completely fixed and market equilibrium will be achieved by quantity constraints on the non-numeraire commodities. In the long run we allow for price flexibility by introducing a so-called "flexibility" parameter $\alpha \in[0,1)$, describing the maximal possible relative decrease or increase of a price relative to $\tilde{p}$. For a given vector $\tilde{p}$ of fixed prices and a flexibility parameter $\alpha$, the set of admissible price systems is given by the set $P(\alpha)$ defined by

$$
P(\alpha)=\left\{p \in \mathbb{R}_{+}^{n+1} \mid(1-\alpha) \tilde{p}_{j} \leq p_{j} \leq(1-\alpha)^{-1} \widetilde{p}_{j}, \forall j \in I_{n}, \text { and } p_{n+1}=1\right\} .
$$


For ease of notation, in the following we define, for $\alpha \in[0,1), \underline{p}_{j}(\alpha)=(1-\alpha) \tilde{p}_{j}, \forall j \in I_{n}$, $\bar{p}_{j}(\alpha)=(1-\alpha)^{-1} \widetilde{p}_{j}, \forall j \in I_{n}$, and $\underline{p}_{n+1}(\alpha)=\bar{p}_{n+1}(\alpha)=1$, so that $P(\alpha)=\left\{p \in \mathbb{R}_{+}^{n+1}\right.$ $\left.\underline{p}_{j}(\alpha) \leq p_{j} \leq \bar{p}_{j}(\alpha), \forall j \in I_{n+1}\right\}$. It is possible to interprete $\alpha$ as a time parameter, in which case $P(\alpha)$ then denotes the set of possible price systems at time $\alpha$. Clearly, we have that $P(0)=\{\widetilde{p}\}$ and $\cup_{\alpha \in[0,1)} P(\alpha)=\left\{p \in \mathbb{R}_{++}^{n+1} \mid p_{n+1}=1\right\}$, meaning that the set of admissible price systems varies from the unique vector of fixed prices $\tilde{p}$ for $\alpha=0$ to the set of all positive prices with the price of the numeraire normalized to one for $\alpha=1$. The definition of the sets $P(\alpha)$ could be generalized considerably while our results would still hold. In fact, it would be sufficient to assume that, for every $j \in I_{n}, \underline{p}_{j}(\alpha)$ and $\bar{p}_{j}(\alpha)$ are continuous function of $\alpha, \underline{p}_{j}(0)=\widetilde{p}_{j}, \bar{p}_{j}(0)=\widetilde{p}_{j}, \lim _{\alpha \uparrow 1} \underline{p}_{j}(\alpha)=0, \lim _{\alpha \uparrow 1} \bar{p}_{j}(\alpha)=\infty$ and $\underline{p}_{n+1}(\alpha)=\bar{p}_{n+1}(\alpha)=1$, $\forall \alpha \in[0,1)$. Again, $P(\alpha)=\left\{p \in \mathbb{R}_{+}^{n+1} \mid \underline{p}_{j}(\alpha) \leq p_{j} \leq \bar{p}_{j}(\alpha), \forall j \in I_{n+1}\right\}$.

The following assumptions with respect to the economy $\mathcal{E}$ are made:

A1. For every consumer $i \in I_{m}$, the consumption set $X^{i}$ belongs to $\mathbb{R}_{+}^{n+1}$, is closed and convex, and $X^{i}+\mathbb{R}_{+}^{n+1} \subset X^{i}$.

A2. For every consumer $i \in I_{m}$, the preference preordering $\succeq^{i}$ on $X^{i}$ is complete, continuous, strongly monotonic, and strongly convex.

A3. For every consumer $i \in I_{m}$, the vector of initial endowments $w^{i}$ belongs to the interior of $X^{i}$.

Notice that the assumption of strong convexity in A2 allows us to work with demand functions instead of demand correspondences.

In general the short-term vector $\tilde{p}$ of fixed prices will not be equal to the price system in any Walrasian equilibrium of the economy $\mathcal{E}$, being a price system $p^{*} \in \mathbb{R}^{n+1}$ and consumption bundles $x^{* i} \in X^{i}, \forall i \in I_{m}$, such that both $\sum_{i=1}^{m} x^{* i}=\sum_{i=1}^{m} w^{i}$ and $x^{* i}$ is a best element for $\succeq^{i}$ in the budget set $\left\{x^{i} \in X^{i} \mid p^{*^{\top}} x^{i} \leq p^{* \top} w^{i}\right\}$ for every $i \in I_{m}$. To equilibrate the demand and the supply under price rigidities one may introduce an equilibrium concept involving vectors of quantity constraints on the net demands and the net supplies of the non-numeraire commodities. Given a price system $p \in \mathbb{R}_{+}^{n+1}$, a rationing scheme on supply $l \in-\mathbb{R}_{+}^{n}$ and a rationing scheme on demand $L \in \mathbb{R}_{+}^{n}$, the constrained budget set of consumer $i \in I_{m}$ is given by

$$
B^{i}(p, l, L)=\left\{x^{i} \in X^{i} \mid p^{\top} x^{i} \leq p^{\top} w^{i} \text { and } l_{j} \leq x_{j}^{i}-w_{j}^{i} \leq L_{j}, \forall j \in I_{n}\right\} .
$$

Following the fix-price literature (see for a recent survey Bénassy [1]), it is assumed that there is no rationing on the market of the numeraire commodity. The corresponding constrained demand $d^{i}(p, l, L)$ of consumer $i$ is defined as the best element for $\succeq^{i}$ in $B^{i}(p, l, L)$. Because of the strong convexity and strong monotonicity assumptions this element is $u^{-}$ nique and lies on the budget hyperplane, i.e., $p^{\top} d^{i}(p, l, L)=p^{\top} w^{i}$. 
Following Drèze [6], for given $\alpha \in[0,1)$, a Drèze equilibrium with respect to the set $P(\alpha)$ of admissible price systems is defined as follows.

\section{Definition 2.1 (Drèze equilibrium)}

For given $\alpha \in[0,1)$, a Drèze equilibrium with respect to the set $P(\alpha)$ (denoted $\left.\mathrm{DE}_{\alpha}\right)$ for the economy $\mathcal{E}=\left(\left\{X^{i}, \succeq^{i}, w^{i}\right\}_{i=1}^{m}, \tilde{p}\right)$ is a price system $p^{*} \in P(\alpha)$, a rationing scheme $\left(l^{*}, L^{*}\right) \in-\mathbb{R}_{+}^{n} \times \mathbb{R}_{+}^{n}$, and, for every consumer $i \in I_{m}$, a consumption bundle $x^{* i} \in X^{i}$ such that

(i) for all $i \in I_{m}, x^{* i}=d^{i}\left(p^{*}, l^{*}, L^{*}\right)$;

(ii) $\quad \sum_{i=1}^{m} x^{* i}=\sum_{i=1}^{m} w^{i}$;

(iii) for all $j \in I_{n}, x_{j}^{* h}-w_{j}^{h}=l_{j}^{*}$ for some $h \in I_{m}$ implies $x_{j}^{* i}-w_{j}^{i}<L_{j}^{*}, \forall i \in I_{m}$, and $x_{j}^{* h}-w_{j}^{h}=L_{j}^{*}$ for some $h \in I_{m}$ implies $x_{j}^{* i}-w_{j}^{i}>l_{j}^{*}, \forall i \in I_{m}$;

(iv) for all $j \in I_{n}, p_{j}^{*}>\underline{p}_{j}(\alpha)$ implies $l_{j}^{*}<x_{j}^{* i}-w_{j}^{i}, \forall i \in I_{m}$, and $p_{j}^{*}<\bar{p}_{j}(\alpha)$ implies $L_{j}^{*}>x_{j}^{* i}-w_{j}^{i}, \forall i \in I_{m}$.

The rationing schemes on supply and demand are assumed to be uniform, i.e., the same for each consumer. This assumption can be relaxed easily. Condition (i) requires that the consumption of each consumer equals his constrained demand, while Condition (ii) is the market clearing condition. Condition (iii) implies that there is not simultaneously rationing on both sides of a market. Condition (iv) precludes supply rationing on the market of some commodity as long as its price is not minimal, whereas demand rationing on the market of a commodity does not take place if its price is not maximal. In case $\alpha=0$, Condition (iv) is redundant. For $\alpha>0$ Condition (iii) is implied by Condition (iv).

A constrained equilibrium without rationing yields a Walrasian equilibrium. It will be shown in Lemma 3.3 that for $\alpha$ large enough it holds that any $\mathrm{DE}_{\alpha}$ is a Walrasian equilibrium. In this paper a price and quantity adjustment process will be considered that starts with the vector of fixed prices $\widetilde{p}$ and an arbitrary rationing scheme $(l, L) \in-\mathbb{R}_{+}^{n} \times \mathbb{R}_{+}^{n}$. In a first stage of quantity adjustments, the initial rationing scheme is adjusted until a shortterm $\mathrm{DE}_{0}$ for the vector of fixed prices $\tilde{p}$ is reached. In the second stage prices are adjusted while markets are kept in equilibrium by infinitesimally small changes in the rationing scheme. In this way the process will generate a path of $\mathrm{DE}_{\alpha}$ 's for varying values of $\alpha$ and will finally converge to a Walrasian equilibrium. At any point on the second stage path all markets are in equilibrium and hence trade is possible. According to Condition (iv) we have that at any equilibrium along the path the price of a commodity $j \in I_{n}$ is on its lower bound $\underline{p}_{j}(\alpha)$ as long as at least one consumer is rationed on his supply of this commodity, the price of a commodity $j$ is on its upper bound $\bar{p}_{j}(\alpha)$ as long as at least one 
consumer is rationed on his demand of this commodity, and the price of a commodity $j$ is allowed to vary between its lower bound $\underline{p}_{j}(\alpha)$ and upper bound $\bar{p}_{j}(\alpha)$ as soon as there is no rationing on this market. So, prices of commodities on markets with supply rationing (demand rationing) are decreased (increased) maximally.

\section{The reduced total excess demand function}

To describe the price and quantity adjustment process, we relate to any element $q$ of the $(n+1)$-dimensional set $C^{n+1}$ given by

$$
C^{n+1}=\left\{q \in \mathbb{R}^{n+1} \mid 0 \leq q_{j} \leq 1, \forall j \in I_{n}, 0 \leq q_{n+1}<1\right\},
$$

a flexibility parameter $\widehat{\alpha}(q)$ and thereby a set $P(\widehat{\alpha}(q))$ of admissible price systems, a price system $\widehat{p}(q) \in P(\widehat{\alpha}(q))$ and a rationing scheme $(\widehat{l}(q), \widehat{L}(q))$. For $q \in C^{n+1}$, define

$$
\begin{aligned}
\widehat{\alpha}(q) & =q_{n+1} \\
\hat{p}_{n+1}(q) & =1,
\end{aligned}
$$

for all $j \in I_{n}$,

$$
\begin{aligned}
\widehat{p}_{j}(q) & =\max \left\{\underline{p}_{j}(\widehat{\alpha}(q)), \min \left\{\left(2-3 q_{j}\right) \underline{p}_{j}(\widehat{\alpha}(q))+\left(3 q_{j}-1\right) \bar{p}_{j}(\widehat{\alpha}(q)), \bar{p}_{j}(\widehat{\alpha}(q))\right\}\right\} \\
\widehat{l}_{j}(q) & =-\min \left\{1,3 q_{j}\right\} w_{j} \\
\widehat{L}_{j}(q) & =\min \left\{1,3-3 q_{j}\right\} \frac{\widetilde{p}^{\top} w}{\widetilde{p}_{j}} .
\end{aligned}
$$

Let some $q \in C^{n+1}$ be given. For every $j \in I_{n}$ we have that $q_{j}=0$ implies $\widehat{l}_{j}(q)=0$ and $q_{j}=1$ implies $\widehat{L}_{j}(q)=0$. Moreover, $0 \leq q_{j}<\frac{1}{3}$ implies $\widehat{p}_{j}(q)=\underline{p}_{j}(\widehat{\alpha}(q)), \hat{l}_{j}(q)>-w_{j}$ and $\widehat{L}_{j}(q)=\frac{\tilde{p}^{\top} w}{\widetilde{p}_{j}}$. Furthermore, $\frac{1}{3} \leq q_{j} \leq \frac{2}{3} \operatorname{implies} \underline{p}_{j}(\widehat{\alpha}(q)) \leq \widehat{p}_{j}(q) \leq \bar{p}_{j}(\widehat{\alpha}(q)), \widehat{l}_{j}(q)=-w_{j}$ and $\widehat{L}_{j}(q)=\frac{\tilde{p}^{\top} w}{\widetilde{p}_{j}}$, whereas $\frac{2}{3}<q_{j} \leq 1$ implies $\widehat{p}_{j}(q)=\bar{p}_{j}(\widehat{\alpha}(q)), \widehat{l}_{j}(q)=-w_{j}$, and $\widehat{L}_{j}(q)<$ $\frac{\tilde{p}^{\top} w}{\widetilde{p}_{j}}$. For any $q$ it holds that $\widehat{p}(q) \in P(\widehat{\alpha}(q))$ since $\widehat{p}_{n+1}(q)=1$.

The properties described in the previous paragraph imply that if $\frac{1}{3} \leq q_{j} \leq 1$, then the rationing scheme on supply on the market of commodity $j, \hat{l}_{j}(q)$, is non-binding for any consumer. Similarly, if $0 \leq q_{j} \leq \frac{2}{3}$ and $q_{n+1}=0$, so $\widehat{\alpha}(q)=0$ and $\widehat{p}(q)=\tilde{p}$, then the rationing scheme on demand on the market of commodity $j, \widehat{L}_{j}(q)$, is not binding for any consumer. Moreover, since $\frac{\tilde{p}^{\top} w}{\widetilde{p}_{j}}>w_{j}$, it is easily seen that $0 \leq q_{j} \leq \frac{2}{3}$ implies that the rationing scheme on the market of commodity $j, \widehat{L}_{j}(q)$, cannot be binding for any consumer in any $\mathrm{DE}_{\widehat{\alpha}(q)}$.

For $q \in C^{n+1}$ we call $\widehat{B}^{i}(q)=B^{i}(\widehat{p}(q), \widehat{l}(q), \widehat{L}(q))$ the reduced constrained budget set of consumer $i \in I_{m}$ at $q$, i.e.,

$$
\widehat{B}^{i}(q)=\left\{x^{i} \in X^{i} \mid \widehat{p}(q)^{\top} x^{i} \leq \widehat{p}(q)^{\top} w^{i} \text { and } \widehat{l}_{j}(q) \leq x_{j}^{i}-w_{j}^{i} \leq \widehat{L}_{j}(q), \forall j \in I_{n}\right\} .
$$


Let $\widehat{d}^{2}(q)$ denote the best element for $\succeq^{i}$ in the reduced constrained budget set $\widehat{B}^{i}(q)$ of consumer $i \in I_{m}$, so $\widehat{d}^{l}(q)=d^{i}(\widehat{p}(q), \widehat{l}(q), \widehat{L}(q))$, let $\widehat{d}(q)=\left(\widehat{d}^{1}(q), \ldots, \widehat{d}^{m}(q)\right)$ denote the collection of vectors of resulting demands, and define the total excess demand at $q$ by

$$
\widehat{z}(q)=\sum_{i=1}^{m} \widehat{d}(q)-\sum_{i=1}^{m} w^{i}
$$

The function $\widehat{z}: C^{n+1} \rightarrow \mathbb{R}^{n+1}$ is called the reduced total excess demand function. For $q^{*} \in C^{n+1}$ it holds that $\left(\widehat{p}\left(q^{*}\right), \widehat{l}\left(q^{*}\right), \widehat{L}\left(q^{*}\right), \widehat{d}\left(q^{*}\right)\right)$ is a $\operatorname{DE}_{\widehat{\alpha}\left(q^{*}\right)}$ if and only if $\widehat{z}\left(q^{*}\right)=\underline{0}$.

Before we describe the process in detail, we describe some properties of the reduced total excess demand function $\widehat{z}$ in the following lemmas.

\section{Lemma 3.1}

Let the economy $\mathcal{E}=\left(\left\{X^{i}, \succeq^{i}, w^{i}\right\}_{i=1}^{m}, \tilde{p}\right)$ satisfy the Assumptions A1-A3. Then the reduced total excess demand function $\widehat{z}$ is continuous on $C^{n+1}$ and $\widehat{p}(q)^{\top} \widehat{z}(q)=0, \forall q \in C^{n+1}$.

\section{Proof}

By the lemma in Drèze [6] (p. 304) it follows that, for every $i \in I_{m}, B^{i}$ is continuous on $\mathbb{R}_{+}^{n} \times\{1\} \times-\mathbb{R}_{+}^{n} \times \mathbb{R}_{+}^{n}$, using that $p_{n+1}=1$ and the absence of rationing on the market of the numeraire commodity. Using the continuity and the strong convexity of the preferences and the maximum theorem it follows that, for every $i \in I_{m}$, $d^{i}$ is continuous on $\mathbb{R}_{+}^{n} \times\{1\} \times-\mathbb{R}_{+}^{n} \times \mathbb{R}_{+}^{n}$. By the continuity of the functions $\widehat{\alpha}, \hat{p}, \hat{l}$ and $\widehat{L}$ in $q$ it follows that $\widehat{z}$ is continuous on $C^{n+1}$. The strong monotonicity of the preferences yields that $\widehat{p}(q)^{\top} \widehat{z}(q)=0, \forall q \in C^{n+1}$.

Q.E.D.

The next lemma gives the behaviour of $\widehat{z}_{j}(q)$ on $C^{n+1}$ for values of $q_{j}, j \in I_{n}$, equal to zero or one.

\section{Lemma 3.2}

Let the economy $\mathcal{E}=\left(\left\{X^{i}, \succeq^{i}, w^{i}\right\}_{i=1}^{m}, \widehat{p}\right)$ satisfy the Assumptions A1-A3. Then, for every $q \in C^{n+1}$, for every $j \in I_{n}, q_{j}=0$ implies $\widehat{z}_{j}(q) \geq 0$ and $q_{j}=1$ implies $\widehat{z}_{j}(q) \leq 0$.

\section{Proof}

If $q_{j}=0$ for some $j \in I_{n}$, then $\widehat{l}_{j}(q)=0$ and hence $\widehat{z}_{j}(q)=\sum_{i=1}^{m}\left(\widehat{d}_{j}^{i}(q)-w_{j}^{i}\right) \geq$ $m \widehat{l}_{j}(q)=0$. Analogously, if $q_{j}=1$ for some $j \in I_{n}$, then $\widehat{L}_{j}(q)=0$, and hence $\widehat{z}_{j}(q)=\sum_{i=1}^{m}\left(\widehat{d}_{j}^{i}(q)-w_{j}^{i}\right) \leq m \widehat{L}_{j}(q)=0$.

Lemma 3.2 will be used to show that the adjustment process will not hit the boundary of $C^{n+1}$ where $q_{j}=0$ or $q_{j}=1$ for some $j \in I_{n}$.

If $q^{*} \in C^{n+1}$ satisfies $q_{n+1}^{*}=0$ and $\widehat{z}\left(q^{*}\right)=\underline{0}$, then $\widehat{p}\left(q^{*}\right)=\widetilde{p}$ and $\left(\widehat{p}\left(q^{*}\right), \widehat{l}\left(q^{*}\right), \widehat{L}\left(q^{*}\right)\right.$, $\left.\widehat{d}\left(q^{*}\right)\right)$ is a $\mathrm{DE}_{0}$. The next lemma states that if $q^{*} \in C^{n+1}$ is such that $\widehat{\alpha}\left(q^{*}\right)=q_{n+1}^{*}$ 
is sufficiently close to one and $\widehat{z}\left(q^{*}\right)=\underline{0}$, then $\left(\widehat{p}\left(q^{*}\right), \widehat{l}\left(q^{*}\right), \widehat{L}\left(q^{*}\right), \widehat{d}\left(q^{*}\right)\right)$ is a Walrasian equilibrium, i.e., for every consumer $i \in I_{m}$ it holds that $\widehat{l}_{j}\left(q^{*}\right)<\widehat{d}_{j}^{i}\left(q^{*}\right)-w_{j}^{i}<\widehat{L}_{j}\left(q^{*}\right)$.

\section{Lemma 3.3}

Let the economy $\mathcal{E}=\left(\left\{X^{i}, \succeq^{i}, w^{i}\right\}_{i=1}^{m}, \widetilde{p}\right)$ satisfy the Assumptions A1-A3. Then there exists $\beta \in(0,1)$ such that for every $q^{*} \in C^{n+1}$ satisfying $\widehat{z}\left(q^{*}\right)=\underline{0}$ and $q_{n+1}^{*} \geq \beta$ it holds that $\left(\widehat{p}\left(q^{*}\right), \widehat{l}\left(q^{*}\right), \widehat{L}\left(q^{*}\right), \widehat{d}\left(q^{*}\right)\right)$ is a WE.

\section{Proof}

First, suppose there exists $\alpha^{*} \in(0,1)$ such that $q_{n+1}^{*}<\alpha^{*}$ for any zero point $q^{*} \in C^{n+1}$ of $\widehat{z}$. Then the lemma is true for $\beta=\alpha^{*}$.

Second, consider the case that there is a sequence $\left(q^{r}\right)_{r \in \mathbb{N}}$ such that for every $r \in \mathbb{N}$, $q_{n+1}^{r} \geq \frac{r-1}{r}$ and $\widehat{z}\left(q^{r}\right)=\underline{0}$. Now, suppose the lemma is not true. Then for all $r \in \mathbb{N}$, $\left(\widehat{p}\left(q^{r}\right), \widehat{l}\left(q^{r}\right), \widehat{L}\left(q^{r}\right), \widehat{d}\left(q^{r}\right)\right)$ is a $\mathrm{DE}_{\widehat{\alpha}\left(q^{r}\right)}$ but not a WE. Modify the consumption set $X^{i}$, $\forall i \in I_{m}$, by imposing the constraint $x_{n+1}^{i} \leq w_{n+1}^{i}+w_{n+1}$. Since $q^{r}$ induces a $\mathrm{DE}_{\hat{\alpha}\left(q^{r}\right)}$, $\widehat{d^{i}}\left(q^{r}\right), \forall i \in I_{m}$, satisfies this constraint with inequality. Without loss of generality, it can be assumed that (i) there is a commodity $j^{\prime} \in I_{n}$ such that $\widehat{p}_{j^{\prime}}\left(q^{r}\right)=\bar{p}_{j^{\prime}}\left(\widehat{\alpha}\left(q^{r}\right)\right), \forall r \in \mathbb{N}$, or (ii) there is a commodity $j^{\prime} \in I_{n}$ such that $\widehat{p}_{j^{\prime}}\left(q^{r}\right)=\underline{p}_{j^{\prime}}\left(\widehat{\alpha}\left(q^{r}\right)\right), \forall r \in \mathbb{N}$, since otherwise there could be no binding rationing in the $\mathrm{DE}_{\widehat{\alpha}\left(q^{r}\right)}$ induced by $q^{r}$ by Condition (iv), implying that $q^{r}$ was a Walrasian equilibrium, contradicting our supposition. Consider the sequence

$$
\left(\frac{\widehat{p}\left(q^{r}\right)}{\left\|\widehat{p}\left(q^{r}\right)\right\|_{\infty}}, \widehat{l}\left(q^{r}\right), \widehat{L}\left(q^{r}\right), \widehat{d}\left(q^{r}\right)\right)_{r \in \mathbb{N}},
$$

which is bounded and therefore, without loss of generality, can be assumed to converge to some $\left(p^{\prime}, l^{\prime}, L^{\prime}, d^{\prime}\right) \in \mathbb{R}_{+}^{n+1} \times-\mathbb{R}_{+}^{n} \times \mathbb{R}_{+}^{n} \times \prod_{i=1}^{m} \mathbb{R}_{+}^{n+1}$. Notice that Condition (ii) of Definition 2.1 implies that $\sum_{i \in I_{m}} d^{i}=\sum_{i \in I_{m}} w^{i}$. Clearly, there is $k \in I_{n+1}$ such that $p_{k}^{\prime}=1$. It follows easily that $l_{k}^{\prime}=-w_{k}$ if $k \in I_{n}$. Therefore, even in case $k=n+1$, $p^{\top^{\top}}\left(l^{\top},-w_{n+1}\right)^{\top}<0$, and so the function $d^{i}, \forall i \in I_{m}$, is continuous at $\left(p^{\prime}, l^{\prime}, L^{\prime}\right)$ by the lemma in Drèze [6] (p. 304). In case (i) it holds that $p_{n+1}^{\prime}=\lim _{r \rightarrow \infty} \frac{\widehat{p}_{n+1}\left(q^{r}\right)}{\left\|\left(q^{r}\right)\right\|_{\infty}}=0$, so by the strict monotonicity of preferences, for every $i \in I_{m}$,

$$
d_{n+1}^{r_{i}}=\lim _{r \rightarrow \infty} \widehat{d}_{n+1}^{i}\left(q^{r}\right)=\lim _{r \rightarrow \infty} d_{n+1}^{i}\left(\widehat{p}\left(q^{r}\right), \widehat{l}\left(q^{r}\right), \widehat{L}\left(q^{r}\right)\right)=d_{n+1}^{i}\left(p^{\prime}, l^{\prime}, L^{\prime}\right)=w_{n+1}^{i}+w_{n+1},
$$

so $w_{n+1}=\sum_{i \in I_{m}} d_{n+1}^{i i}=(m+1) w_{n+1}$, a contradiction. In case (ii) it holds that $p_{j^{\prime}}^{\prime}=0$ and $L_{j^{\prime}}^{\prime}=\frac{\tilde{p}^{\top} w}{\widetilde{p}_{j^{\prime}}}$, so by the strict monotonicity of preferences, for every $i \in I_{m}$,

$$
\begin{aligned}
d_{j^{\prime}}^{i} & =\lim _{r \rightarrow \infty} \widehat{d}_{j^{\prime}}^{i}\left(q^{r}\right)=\lim _{r \rightarrow \infty} d_{j^{\prime}}^{i}\left(\widehat{p}\left(q^{r}\right), \widehat{l}\left(q^{r}\right), \widehat{L}\left(q^{r}\right)\right) \\
& =d_{j^{\prime}}^{i}\left(p^{\prime}, l^{\prime}, L^{\prime}\right)=w_{j^{\prime}}^{i}+\frac{\widehat{p}^{\top} w}{\widetilde{p}_{j^{\prime}}}>w_{j^{\prime}}^{i}+w_{j^{\prime}},
\end{aligned}
$$


so $w_{j^{\prime}}=\sum_{i \in I_{m}} d_{j^{\prime}}^{\prime i}>(m+1) w_{j^{\prime}}$, a contradiction. Consequently, the lemma is true. Q.E.D.

For the remainder of the paper we fix a real number $\bar{\beta} \in(0,1)$, satisfying the requirement of Lemma 3.3, and define the sets $\widehat{C}^{n+1}, \underline{C}^{n+1}$, and $\bar{C}^{n+1}$ by $\widehat{C}^{n+1}=\left\{q \in C^{n+1} \mid q_{n+1} \leq \bar{\beta}\right\}$, $\underline{C}^{n+1}=\left\{q \in C^{n+1} \mid q_{n+1}=0\right\}$ and $\bar{C}^{n+1}=\left\{q \in C^{n+1} \mid q_{n+1}=\bar{\beta}\right\}$. In Section 5 we give a constructive proof of the existence of a path of points in $\widehat{C}^{n+1}$ leading from an arbitrarily chosen initial state $v \in \underline{C}^{n+1}$ to an approximate zero point $q^{*} \in \bar{C}^{n+1}$ of $\widehat{z}$, which therefore induces an approximate Walrasian equilibrium.

\section{Definition and Illustration of the Price and Quan- tity Adjustment Process}

In this section an initial state $v$ in the relative interior of $\underline{C}^{n+1}$ is assumed to be given. Notice that the initial state $v$ induces a flexibility parameter $\widehat{\alpha}(v)=0$, a price system $\widehat{p}(v)=\widetilde{p}$, and a rationing scheme $(\widehat{l}(v), \widehat{L}(v))$. The price and quantity adjustment process is defined and illustrated using the reduced total excess demand function $\widehat{z}$. In the next section it is shown that the price and quantity adjustment process converges to a Walrasian equilibrium, irrespective of the choice of the initial state. The proofs given in that section are given for a piecewise linear approximation of the reduced total excess demand function, where the inaccuracy of the approximation can be made arbitrarily small. The price and quantity adjustment process consist of two parts. First, only quantities, i.e., the rationing schemes, are adjusted and the price system is assumed to be fixed and equal to $\widetilde{p}$. This part of the adjustment process is referred to as the short-term adjustment process. The short-term adjustment process results in a $\mathrm{DE}_{0}$ and coincides with the quantity adjustment process given in Herings [10] to find a Drèze equilibrium given a fixed price system. In that paper it has been shown that for every initial state $v \in \underline{C}^{n+1}$, for almost every economy (where an economy is parametrized by the initial endowments) the quantity adjustment process converges to a uniquely determined $\mathrm{DE}_{0}$. The proofs given there are for the function $\widehat{z}$ itself, but are extremely tedious. Therefore, the proofs given in the next section are related to a piecewise linear approximation of $\widehat{z}$, allowing us to give considerably easier demonstrations. Secondly, after a $\mathrm{DE}_{0}$ has been found, the prices are adjusted and the quantities, i.e., the rationing schemes, are assumed to change in such a way that the markets stay in equilibrium. This part of the adjustment process is referred to as the long-term adjustment process and results in a Walrasian equilibrium of the economy.

More in detail, the behaviour of the price and quantity adjustment process can be described as follows. Initially, from $v$ the path proceeds in the subset $\underline{C}^{n+1}$ of $\widehat{C}^{n+1}$ until a zero point $q^{*} \in \underline{C}^{n+1}$ of $\widehat{z}$ is reached. Clearly, $q^{*}$ induces a $\mathrm{DE}_{0}$ since at $q^{*}$ it holds 
that $\widehat{\alpha}\left(q^{*}\right)=q_{n+1}^{*}=0$. In general, the initial state $v$ is incompatible with a $\mathrm{DE}_{0}$, i.e., $\widehat{z}(v) \neq \underline{0}$. First only adjustments of the rationing scheme take place, being based on the excess demands on the markets of the non-numeraire commodities and on the change of the rationing scheme compared to the initial state, whereas the price system remains initially unchanged. So initially the process generates a path in $\underline{C}^{n+1}$. If there is a negative excess demand on a market, the rationing scheme is adjusted in such a way that, compared with the initial state induced by point $v$, the rationing is tightened maximally in case of supply rationing and is weakened maximally in case of demand rationing. So, compared with the initial state, consumers are enforced to supply less or are allowed to demand more of this commodity. If there is a positive excess demand on a market, the rationing scheme is adjusted in such a way that, compared with the initial state induced by point $v$, the rationing is tightened maximally in case of demand rationing and weakened maximally in case of supply rationing. If a market is in equilibrium then the rationing scheme is adjusted in such a way that the market is kept in equilibrium.

To make this more precise, the properties of the states of the economy reached by this quantity adjustment process can be formulated mathematically as follows. If a point $q \in \underline{C}^{n+1}$ is reached by the process, then there exists a real number $\gamma \in[0,1]$ such that for every $j \in I_{n}$,

$$
\begin{array}{ll}
q_{j}=\gamma v_{j} & \text { if } \widehat{z}_{j}(q)<0, \\
\gamma v_{j} \leq q_{j} \leq 1-\gamma\left(1-v_{j}\right) & \text { if } \widehat{z}_{j}(q)=0, \\
q_{j}=1-\gamma\left(1-v_{j}\right) & \text { if } \widehat{z}_{j}(q)>0 .
\end{array}
$$

Notice that the initial state $v$ satisfies the properties given above for $\gamma=1$. Observe furthermore that these properties are closely related to the ideas behind the Walrasian tatonnement process. In fact, at a point $q$ reached by the proces we have that $\frac{q_{j}}{v_{j}}=$ $\min _{h \in I_{n}}\left\{\frac{q_{h}}{v_{h}}\right\}$ if $\widehat{z}_{j}(q)<0$, so that relative to $v, q_{j}$ is minimal if there is excess supply of commodity $j$. On the other hand we have that $\frac{1-q_{j}}{1-v_{j}}=\min _{h \in I_{n}}\left\{\frac{1-q_{h}}{1-v_{h}}\right\}$ if $\widehat{z}_{j}(q)>0$, so that relative to $v, 1-q_{j}$ is minimal if there is excess demand of commodity $j$. Finally, when $\widehat{z}_{j}(q)=0$, then $q_{j}$ is allowed to vary between these relative lower and upper bounds in order to keep market $j$ in equilibrium. In Herings [10] it is shown that for a generic economy the properties (6) determine the short-term adjustment process, up to the speed of adjustment, uniquely. As a heuristic, notice that the system of equations in (6) has $n+1$ independent free variables, $q_{1}, \ldots, q_{n}$ and $\gamma$, and $n$ equations, leaving one degree of freedom being the dimension of the adjustment path. The process leads to a point $q^{*} \in \underline{C}^{n+1}$, yielding a $\mathrm{DE}_{0}$. Observe that at this point the properties (6) are satisfied for $\gamma=\min _{j \in I_{n}}\left\{\frac{q_{j}^{*}}{v_{j}}, \frac{1-q_{j}^{*}}{1-v_{j}}\right\}$.

Continuing from this point $q^{*}$, the process generates a path of zero points of $\widehat{z}$ in $\widehat{C}^{n+1}$. Along the path the flexibility parameter $\alpha$ is adjusted according to the adjustment of the value of $q_{n+1}$. Moreover, if there is demand (supply) rationing on the market of a 
commodity, then its price is increased (decreased) relatively maximal, whereas the rationing scheme is adjusted such that any point on the path induces a $\mathrm{DE}_{\hat{\alpha}(q)}$. It will be shown that this long-term process of following a path of $\mathrm{DE}_{\hat{\alpha}(q)}$ 's by price and quantity adjustments either comes back to a point in $\underline{C}^{n+1}$ at which $q_{n+1}=0$, or ends at a point in $\bar{C}^{n+1}$ at which $q_{n+1}=\bar{\beta}$. In the latter case a Walrasian equilibrium has been found according to Lemma 3.3. In the first case the process has found a second $D_{0}$. Then the process continues in $\underline{C}^{n+1}$ by short-term adjustments of the rationing scheme, while simultaneously the markets are allowed to become out of equilibrium again. Along this path the rationing scheme is adjusted again according to the excess demands and the position of the rationing scheme with respect to the initial state $v$, until a new (third) $\mathrm{DE}_{0}$ is found. From this point on the process continues again in $\widehat{C}^{n+1}$ by following a path of $\mathrm{DE}_{\widehat{\alpha}(q)}$ 's. It will be shown that eventually a $\mathrm{DE}_{0}$ will be reached from where the long-term path of $\mathrm{DE}_{\hat{\alpha}(q)}$ 's leads to a point $q$ in $\bar{C}^{n+1}$, i.e., to a Walrasian equilibrium.

Mathematically, the properties of the states of the economy reached by the longterm adjustment process can be formulated as follows. If $q^{*} \in \widehat{C}^{n+1}$ is reached by the long-term adjustment process, then

$$
\widehat{z}\left(q^{*}\right)=\underline{0},
$$

so $q^{*}$ induces a $\mathrm{DE}_{\widehat{\alpha}\left(q^{*}\right)}$. Again, it should be expected that for a generic economy property (7) determines uniquely the long-term adjustment process, up to the speed of adjustment, uniquely. As a heuristic, notice that the system of equations in (7) has $n+1$ independent free variables, $q_{1}, \ldots, q_{n+1}$, and, by Walras law, $n$ independent equations, leaving one degree of freedom being the dimension of the adjustment path. We will not give a formal proof of this claim, but we will show in the next section that the long-term adjustment process is uniquely determined for any non-degenerate piecewise linear approximation of the reduced total excess demand function, where the inaccuracy of the approximation can be made arbitrarily small. Observe that according to Lemma 3.3 any $q^{*} \in \widehat{C}^{n+1}$ with $\widehat{\alpha}\left(q^{*}\right)=$ $q_{n+1}^{*}=\bar{\beta}$ satisfying property $(7)$ is a Walrasian equilibrium.

By definition of the reduced total excess demand function $\widehat{z}$ along the path determined by property ( 7 ) it holds that, for $j \in I_{n}$, the prices satisfy the conditions

$$
\begin{array}{ll}
\widehat{p}_{j}\left(q^{*}\right)=\left(1-\widehat{\alpha}\left(q^{*}\right)\right) \tilde{p}_{j} & \text { if } q_{j}^{*} \leq \frac{1}{3}, \\
\left(1-\widehat{\alpha}\left(q^{*}\right)\right) \widetilde{p}_{j} \leq \widehat{p}_{j}\left(q^{*}\right) \leq\left(1-\widehat{\alpha}\left(q^{*}\right)\right)^{-1} \widetilde{p}_{j} & \text { if } \frac{1}{3} \leq q_{j}^{*} \leq \frac{2}{3}, \\
\widehat{p}_{j}\left(q^{*}\right)=\left(1-\widehat{\alpha}\left(q^{*}\right)\right)^{-1} \widetilde{p}_{j} & \text { if } q_{j}^{*} \geq \frac{2}{3} .
\end{array}
$$

Therefore, along the path of long-term price and quantity adjustments, the price of a commodity is kept on its lower bound with respect to $\widehat{\alpha}\left(q^{*}\right)$ as long as there is supply rationing on the market of this commodity and the price of a commodity is kept on its upper bound with respect to $\widehat{\alpha}\left(q^{*}\right)$ as long as there is demand rationing on the market 
of this commodity. To keep the market in equilibrium, the price of a commodity may vary between these lower and upper bounds if there is no rationing on the market of this commodity. Notice that these properties are again closely related to the ideas behind the Walrasian tatonnement process.

It will be shown in the next section that the long-term adjustment process eventually will reach a Walrasian equilibrium.

Let $Q$ denote the subset of $\widehat{C}^{n+1}$ whose points satisfy (6) or (7), i.e., all points $q$ satisfying the properties of points generated by the price and quantity adjustment process. Not all the points in the set $Q$ will actually be reached by the adjustment process. This will only hold for the points in $Q$ which are connected to the starting point $v$. Denote the component of $v$ in $Q$ by $Q_{v}$. As has been argued above the set $Q$, and also its component containing $v$, can be expected to be 1-dimensional in general. This leads us to the following definition.

\section{Definition 4.1 (Price and quantity adjustment process)}

The price and quantity adjustment process for the economy $\mathcal{E}=\left(\left\{X^{i}, \succeq^{i}, w^{i}\right\}_{i=1}^{m}, \tilde{p}\right)$ with starting point $v \in \underline{C}^{n+1}$ is given by the set $Q_{v}$, the component of $v$ in $Q$.

Notice that the price and quantity adjustment process is now defined by considering explicitly the points $q$ generated by it. Since we have given a topological definition of the process, we will also give a topological definition of the convergence of it.

\section{Definition 4.2 (Convergence)}

The price and quantity adjustment process for the economy $\mathcal{E}=\left(\left\{X^{i}, \succeq^{i}, w^{i}\right\}_{i=1}^{m}, \tilde{p}\right)$ with starting point $v \in \underline{C}^{n+1}$ is convergent if $Q_{v}$ is an arc, i.e. a set homeomorphic to the unit interval [0,1], having $v$ and a Walrasian equilibrium price system as its boundary points.

Notice that any arc described as the zero points of a system of continuously differentiable functions satisfying certain regularity properties can be described by a system of differential equations, see for instance Garcia and Zangwill [8]. Hence, by making suitable differentiability and regularity conditions, it is possible to express the adjustment process in a more standard way by means of a system of differential equations. This is also the approach as adopted by Smale [26] and Kamiya [13]. In this paper we will not make any differentiability assumptions. However, we will provide a method to follow the adjustment process arbitrarily close, even in the case where the function $\widehat{z}$ is only continuous.

We conclude this section with an example to illustrate the process. Therefore we take an economy with two consumers and three commodities. Let the consumption sets of the two consumers be given by $X^{i}=\mathbb{R}_{+}^{n+1}, i=1,2$, and let their preferences be represented by the utility functions $u^{i}: X^{i} \rightarrow \mathbb{R}$ defined by $u^{i}\left(x^{i}\right)=x_{1}^{i} x_{2}^{i} x_{3}^{i}, i=1,2$. The 
initial endowments are given by $w^{1}=(4,0,1)^{\top}$ and $w^{2}=(0,2,1)^{\top}$. Finally, $\tilde{p}$ is given by $\tilde{p}_{j}=2, j=1,2$, and $\tilde{p}_{j}=1$ for the numeraire commodity $j=3$. So, $\tilde{p}^{\top} w=14$ is the value of the total initial endowments at price system $\tilde{p}$. Observe that the Walrasian equilibrium price system is given by $p^{*}=\left(\frac{1}{2}, 1,1\right)^{\top}$.

Now, suppose the initial point $v \in \underline{C}^{3}$ is given by $v=\left(\frac{1}{2}, \frac{1}{2}, 0\right)^{\top}$. So in the initial state there is no binding rationing for any consumer on any market. According to equations (1)-(5) we have that $\widehat{\alpha}(v)=0, \widehat{p}(v)=(2,2,1)^{\top}, \widehat{l}(v)=(-4,-2)^{\top}$ and $\widehat{L}(v)=(7,7)^{\top}$. From this it follows that $\widehat{z}(v)=\left(-\frac{5}{3}, \frac{1}{3}, \frac{8}{3}\right)^{\top}$. So, at $v$ there is excess supply of the first commodity and excess demand of the second commodity. For increasing values of $\gamma$ the process now follows a path of points $q \in \underline{C}^{3}$ such that $q_{1}=\gamma v_{j}=\frac{1}{2} \gamma$ and $q_{2}=1-\gamma\left(1-v_{j}\right)=1-\frac{1}{2} \gamma$, until a point $q$ is reached at which one of the markets reaches an equilibrium. This happens for $\gamma^{1}=\frac{11}{36}$ at the point $q^{1}$ with $q_{1}^{1}=\frac{11}{72}$ and $q_{2}^{1}=\frac{61}{72}$, inducing the rationing scheme $\widehat{l}\left(q^{1}\right)=\left(-\frac{11}{6},-2\right)^{\top}$ and $\widehat{L}\left(q^{1}\right)=\left(7, \frac{77}{24}\right)^{\top}$. At this rationing scheme the first consumer is rationed on his supply of commodity 1 . His constrained demand is $\widehat{d}^{1}\left(q^{1}\right)=\left(\frac{13}{6}, \frac{7}{6}, \frac{7}{3}\right)^{\top}$. For the second consumer the rationing scheme is not binding. His constrained demand is $\widehat{d}^{2}\left(q^{1}\right)=\left(\frac{5}{6}, \frac{5}{6}, \frac{5}{3}\right)^{\top}$. Hence $\widehat{z}\left(q^{1}\right)=(-1,0,2)^{\top}$ and indeed market 2 is in equilibrium. Now, the process continues by decreasing $q_{2}$ from $\frac{61}{72}$ to $\frac{7}{36}$. At the point $q^{2}=\left(\frac{11}{72}, \frac{7}{36}, 0\right)^{\top} \in \underline{C}^{3}$ we have that $\widehat{l}\left(q^{2}\right)=\left(-\frac{11}{6},-\frac{7}{6}\right)^{\top}$ and $\widehat{L}\left(q^{2}\right)=(7,7)^{\top}$. At this rationing scheme the constrained demands of the consumers are still the same as in the point $q^{1}$, but the rationing on the supply of commodity 2 becomes binding for consumer 2 . The point still satisfies the conditions (6) for $\gamma^{2}=\gamma^{1}=\frac{11}{36}$. Observe that market 2 is still in equilibrium and that the value of $q_{2}^{2}$ is still between its lower and its upper bound. A further decrease of the value of $q_{2}$ reduces the supply of commodity 2 by consumer 2 . Therefore, in order to keep the market of commodity 2 in equilibrium, also the value of $q_{1}$ is further decreased. This reduces the demand of consumer 1 for both commodities 1 and 2. Market 2 is kept in equilibrium by decreasing $q_{1}$ and $q_{2}$ simultaneously along the line $q_{2}=\frac{24 q_{1}+1}{24}$. At the point $q^{3}=\left(\frac{1}{24}, \frac{1}{12}, 0\right)^{\top} \in \underline{C}^{3}$ we have that $\widehat{l}\left(q^{3}\right)=\left(-\frac{1}{2},-\frac{1}{2}\right)^{\top}$ and $\widehat{L}\left(q^{3}\right)=(7,7)^{\top}$. At this rationing scheme the constrained demands of the consumers are equal to $\widehat{d}^{1}\left(q^{3}\right)=\left(\frac{7}{2}, \frac{1}{2}, 1\right)^{\top}$ and $\widehat{d}^{2}\left(q^{3}\right)=\left(\frac{1}{2}, \frac{3}{2}, 1\right)^{\top}$. Hence all markets are in equilibrium and a $\mathrm{DE}_{0}$, where $P(0)=$ $\left\{(2,2,1)^{\top}\right\}$, is obtained by short-term adjustments of the rationing scheme. This path in $C^{3}$ generated by the process is illustrated in Figure 1.

From the point $q^{3}$ the process follows a uniquely determined long-term path of zero points of $\widehat{z}$ in $\widehat{C}^{3}$ satisfying the conditions (8) by adjusting simultaneously the prices and the rationing scheme. At the point $q^{3}$ we have supply rationing on both markets. So, along the long-term path, initially both prices are kept on their lower bound with respect to $P(\widehat{\alpha}(q))$. So, for $q_{3}$ small enough we have for $j=1,2$ that $\widehat{p}_{j}=(1-\widehat{\alpha}(q)) \widetilde{p}_{j}=\left(1-q_{3}\right) \widetilde{p}_{j}$. Increasing $q_{3}$ from zero the process follows the path given by $\left\{q \in \widehat{C}^{n+1} \mid q_{1}=\frac{1}{24\left(1-q_{3}\right)}, q_{2}=\frac{1}{12\left(1-q_{3}\right)}\right\}$, until 
Figure 1: The short-term path

the point $q^{4}=\left(\frac{1}{12}, \frac{1}{6}, \frac{1}{2}\right)^{\top}$ is reached. Going along this line from $q^{3}$ to $q^{4}$ a path of $\operatorname{DE}_{\widehat{\alpha}(q)}$ is followed given by $\widehat{p}_{j}(q)=\left(1-q_{3}\right) \widetilde{p}_{j}=2\left(1-q_{3}\right), j=1,2$, and $\widehat{l}_{j}(q)=-\min \left\{1,3 q_{j}\right\} w_{j}=$ $3 q_{j} w_{j}, j=1,2$. At the point $q^{4}$ we have that $\widehat{p}\left(q^{4}\right)=(1,1,1)^{\top}$ and $\widehat{l}\left(q^{4}\right)=(-1,-1)^{\top}$. The path going from $q^{3}$ to $q^{4}$ is characterized by Keynesian multiplier effects. For instance, a decrease in the price of commodity 1 leads to an increase in the demand of commodity 1 by consumer 2 . Therefore, the rationing of consumer 1 on his supply of commodity 1 is weakened, which leads to more income for consumer 1 and hence more demand of commodity 2 by consumer 1 . Consequently, the rationing of consumer 2 on his supply of commodity 2 is weakened, giving him more income income and hence more demand of commodity 1, etc. These effects are even enforced, since also the price of commodity 2 decreases on this part of the path. However, at prices $p=(1,1,1)^{\top}$ the unconstrained supply of commodity 2 by consumer 2 is equal to 1 and hence the supply constraint on commodity 2 becomes non-binding at the point $q^{4}$. So, from this point on the lower bound on $p_{2}$ becomes non-binding. Therefore, continuing along the path from the point $q^{4}$ the process increases the value of $q_{2}$ until the point $q^{5}=\left(\frac{1}{12}, \frac{1}{3}, \frac{1}{2}\right)$ is reached where it holds that $\hat{p}_{2}\left(q^{5}\right)=\underline{p}_{2}\left(\widehat{\alpha}\left(q^{5}\right)\right)=\left(2-3 q_{2}^{5}\right) \underline{p}_{2}\left(\widehat{\alpha}\left(q^{5}\right)\right)+\left(3 q_{2}^{5}-1\right) \bar{p}_{2}\left(\widehat{\alpha}\left(q^{5}\right)\right)=1<\bar{p}_{2}\left(\widehat{\alpha}\left(q^{5}\right)\right)$, so that the price of commodity 2 exceeds its lower level if the value of $q_{3}$ is increased further. Then indeed the value of $q_{3}$ is increased again, while simultaneously the values of $q_{1}$ and $q_{2}$ are adjusted in order to keep the markets of the commodities 1 and 2 in equilibrium. Along 
this part of the path we have that the price of commodity 1 is on its lower bound and the first market is kept in equilibrium by adjusting the supply constraint on market 1, while the price of commodity 2 is above its lower bound and the second market is kept in equilibrium without rationing by adapting the price. So, for given value $q_{3}$ on this part of the path, let $l_{1}$ be the corresponding equilibrium supply rationing and let $p_{2}$ be the corresponding price of commodity 2. Since $\hat{p}_{1}(q)=\underline{p}_{1}(\widehat{\alpha}(q))=2\left(1-q_{3}\right)$, the current price system for given value of $q_{3}$ is given by $p=\left(2\left(1-q_{3}\right), p_{2}, 1\right)^{\top}$, while consumer 1 is constrained by $l_{1}<0$ on his supply of commodity 1 and consumer 2 is unrationed. So, at a price system $p$ induced by a vector $q$ on this part of the path the reduced demands for the commodities 1 and 2 are given by

$$
d_{1}^{1}=4+l_{1}, d_{2}^{1}=\frac{1-p_{1} l_{1}}{2 p_{2}}=\frac{1-2\left(1-q_{3}\right) l_{1}}{2 p_{2}},
$$

and

$$
d_{1}^{2}=\frac{2 p_{2}+1}{3 p_{1}}=\frac{2 p_{2}+1}{6\left(1-q_{3}\right)}, d_{2}^{2}=\frac{2 p_{2}+1}{3 p_{2}} .
$$

Since supply equals demand it follows that along the path $l_{1}=-\frac{2 p_{2}+1}{6\left(1-q_{3}\right)}$ and $p_{2}=1$. From equations (3) and (4) it then follows that along the path

$$
l_{1}=\widehat{l}_{1}(q)=-12 q_{1}=-\frac{2 p_{2}+1}{6\left(1-q_{3}\right)}=-\frac{1}{2\left(1-q_{3}\right)}
$$

and

$$
p_{2}=\widehat{p}_{2}(q)=\left(2-3 q_{2}\right) 2\left(1-q_{3}\right)+\left(3 q_{2}-1\right) \frac{2}{\left(1-q_{3}\right)}=1
$$

must hold. From this we obtain that for increasing values of $q_{3}$ the process follows the path given by

$$
q_{1}=\frac{1}{24\left(1-q_{3}\right)}, q_{2}=\frac{-4\left(q_{3}\right)^{2}+7 q_{3}-1}{6 q_{3}\left(2-q_{3}\right)} .
$$

The process follows this path until the point $q^{6}=\left(\frac{1}{6}, \frac{16}{45}, \frac{3}{4}\right)^{\top}$ is reached, inducing the Walrasian equilibrium price system $p=\left(\frac{1}{2}, 1,1\right)^{\top}$. At this price system the unconstrained supply of commodity 1 by consumer 1 is equal to 2 and hence the supply constraint $\widehat{l}_{1}\left(q^{6}\right)=$ $-3 q_{1} w_{1}=-2$ becomes indeed not binding at this point. So, a Walrasian equilibrium has been found. The projection of the long-term path in $\widehat{C}^{3}$ on the $\left(q_{1}, q_{2}\right)$-space has been drawn in Figure 2.

As a technical exercise it is possible to continue along the path by solving for $(7)$. The remainder of the path will consist only of $q$ 's inducing Walrasian equilibria. Starting from $q^{6}$ first the value of $q_{1}$ is increased until the point $q^{7}=\left(\frac{1}{3}, \frac{16}{45}, \frac{3}{4}\right)^{\top}$ is reached, where $\widehat{l}\left(q^{7}\right)=(-4,-2)^{\top}$ and $\widehat{L}\left(q^{7}\right)=(7,7)^{\top}$, so all possibilities for rationing are eliminated. 
Figure 2: The projection on the $\left(q_{1}, q_{2}\right)$-space of the long-term path

Next the value of $q_{3}$ is further increased towards 1 , while the values of $q_{1}$ and $q_{2}$ are simply adjusted to guarantee that the prices keep there Walrasian equilibrium values, $\widehat{p}_{1}(q)=\frac{1}{2}$ and $\widehat{p}_{2}(q)=1$. It is easily verified that for $q_{3} \in\left(\frac{3}{4}, 1\right)$ the path is given by

$$
q_{1}=\frac{-8\left(q_{3}\right)^{2}+15 q_{3}-3}{12 q_{3}\left(2-q_{3}\right)}, q_{2}=\frac{-4\left(q_{3}\right)^{2}+7 q_{3}-1}{6 q_{3}\left(2-q_{3}\right)} .
$$

Notice that on this last part of the path there is not any real change occurring, at any point on the path the price system and the rationing scheme stay the same.

\section{The Approximate Price and Quantity Adjustment Process}

In this section attention is focused on the price and quantity adjustment process for a piecewise linear approximation of the reduced total excess demand function, where the inaccuracy of the approximation can be taken arbitrarily small. We show that, under a standard non-degeneracy condition, for any arbitrarily chosen starting point $v$ in the relative interior of $\underline{C}^{n+1}$ there exists a unique path of points connecting $v$ with a point in $\bar{C}^{n+1}$ yielding an approximate Walrasian equilibrium. The first part of the path is in $\underline{C}^{n+1}$ and connects $v$ with a point $q^{* 1} \in \underline{C}^{n+1}$ yielding an approximate $\mathrm{DE}_{0}$. Then the 
process continues with a path in $\widehat{C}^{n+1} \backslash \underline{C}^{n+1}$ such that any point $q$ on the path yields an approximate $\mathrm{DE}_{\widehat{\alpha}(q)}$. It may happen that this part of the path ends in a second approximate $\mathrm{DE}_{0}$ induced by some point $q^{* 2} \in \underline{C}^{n+1}$. Then the process continues in $\underline{C}^{n+1}$ and finds a third approximate $\mathrm{DE}_{0}$, from where the process continues again in $\widehat{C}^{n+1} \backslash \underline{C}^{n+1}$ with any point $q$ on the path inducing an approximate $\mathrm{DE}_{\widehat{\alpha}(q)}$. Eventually the process finds in $\underline{C}^{n+1}$ an approximate $\mathrm{DE}_{0}$ from where the path of approximate $\mathrm{DE}_{\hat{\alpha}(q)}$ 's leads to an approximate Walrasian equilibrium induced by some point in $\bar{C}^{n+1}$. Applying the technique of simplicial approximation provides us both with a constructive proof of the existence of such a path of points and with the possibility to follow this path. By taking the mesh size of the underlying triangulation small enough the inaccuracy of the approximation can be made arbitrarily small. In the following definition an approximate $\mathrm{DE}_{\alpha}$ for any parameter value of $\alpha \in[0,1)$ is introduced.

\section{Definition $5.1\left(\varepsilon-\mathrm{DE}_{\alpha}\right)$}

For given $\alpha \in[0,1)$ and given real number $\varepsilon \geq 0$, an $\varepsilon-\mathrm{DE}_{\alpha}$ for the economy $\mathcal{E}=\left(\left\{X^{i}, \succeq^{i}\right.\right.$, $\left.\left.w^{i}\right\}_{i=1}^{m}, \tilde{p}\right)$ is a price system $p \in P(\alpha)$, a rationing scheme $(l, L) \in-\mathbb{R}_{+}^{n} \times \mathbb{R}_{+}^{n}$ and for every consumer $i \in I_{m}$ a consumption bundle $x^{i} \in X^{i}$ such that all conditions of a $D E_{\alpha}$ are satisfied with the condition of equality of demand and supply replaced by $\| \sum_{i=1}^{m} x^{i}-$ $\sum_{i=1}^{m} w^{i} \|_{\infty} \leq \varepsilon$

Clearly, a $0-\mathrm{DE}_{\alpha}$ is a $\mathrm{DE}_{\alpha}$. In order to show the existence of the described path we will use some techniques of simplicial approximation of functions. We first recall the concept of a simplicial subdivision.

For given $t \in \mathbb{N}, 0 \leq t \leq k$, a $t$-dimensional simplex or $t$-simplex is defined as the convex hull of $t+1$ affinely independent vectors in $\mathbb{R}^{k}, q^{1}, \ldots, q^{t+1}$, and is denoted by $\sigma\left(q^{1}, \ldots, q^{t+1}\right)$ or shortly by $\sigma$. The vectors $q^{1}, \ldots, q^{t+1}$ are called the vertices of $\sigma$. A $(t-1)$-simplex $\tau$ being the convex hull of $t$ vertices of $\sigma\left(q^{1}, \ldots, q^{t+1}\right)$ is called a facet of $\sigma$. For $h \in I_{t+1}$ the facet $\tau\left(q^{1}, \ldots, q^{h-1}, q^{h+1}, \ldots, q^{t+1}\right)$ is called the facet of $\sigma$ opposite the vertex $q^{h}$. For $0 \leq j \leq t$, a $j$-simplex being the convex hull of $j+1$ vertices of a $t$-simplex $\sigma$ is called a face of $\sigma$. A finite collection $\mathcal{T}$ of $k$-simplices is a triangulation of a compact, convex $k$-dimensional subset $S$ of some Euclidean space if:

1. $S$ is the union of all simplices in $\mathcal{T}$;

2. the intersection of two simplices in $\mathcal{T}$ is either empty or a common face of both.

It can be shown that each facet $\tau$ of a $k$-simplex $\sigma \in \mathcal{T}$ either lies in the relative boundary of $S$ and is only a facet of $\sigma$ or it is a facet of exactly one other $k$-simplex in $\mathcal{T}$. The mesh size of a triangulation $\mathcal{T}$ is defined by $\operatorname{mesh}(\mathcal{T})=\max _{\sigma \in \mathcal{T}} \max \left\{\|\widetilde{q}-\widehat{q}\|_{\infty} \mid \widetilde{q}, \widehat{q} \in \sigma\right\}$. 
Let a compact, convex $t$-dimensional subset $S$ of some Euclidean space, a triangulation $\mathcal{T}$ of $S$ and a function $f: S \rightarrow \mathbb{R}^{k}$ be given. A function $F: S \rightarrow \mathbb{R}^{k}$ is called the piecewise linear approximation of $f$ with respect to $\mathcal{T}$ if for every vertex $q$ of any $\sigma \in \mathcal{T}$ it holds that $F(q)=f(q)$ and for every element $q$ of $S$ it holds that $F(q)=\sum_{h \in I_{t+1}} \lambda_{h} F\left(q^{h}\right)$, when $q \in \sigma\left(q^{1}, \ldots, q^{t+1}\right)$ for some $t$-simplex $\sigma \in \mathcal{T}$ and $q=\sum_{h \in I_{t+1}} \lambda_{h} q^{h}$ for some $\lambda \in \mathbb{R}_{+}^{t+1}$ with $\sum_{h \in I_{t+1}} \lambda_{h}=1$.

The price and quantity adjustment process will be considered for a piecewise linear approximation $\widehat{Z}$ of the reduced total excess demand function $\widehat{z}$ with respect to a given triangulation $\mathcal{T}$ of $\widehat{C}^{n+1}$ with an arbitrarily small mesh size. As is intuitively clear, and as will be shown formally later on, a point $q \in \widehat{C}^{n+1}$ satisfying $\widehat{Z}(q)=\underline{0}$ is an approximate $\mathrm{DE}_{\widehat{\alpha}(q)}$ if the inaccuracy of the approximation is small enough. Therefore, such a point $q$ will be called a $\mathrm{DE}_{\widehat{\alpha}(q)}$ of $\widehat{Z}$ or an approximate $\mathrm{DE}_{\widehat{\alpha}(q)}$. The short-term adjustment process operates in the $n$-dimensional subset $\underline{C}^{n+1}$. The obtain the path generated by it one can apply the product-ray algorithm described in Doup and Talman [5] or the exponentray algorithm given in Doup, van den Elzen, and Talman [4] to the function $\widehat{Z}$ on the set $\underline{C}^{n+1}$. Due to the special properties of the function $\widehat{z}$ and therefore of $\widehat{Z}$ we will be able to derive several interesting properties of the path. After the short-term adjustment process has reached a $\mathrm{DE}_{0}$ of $\widehat{Z}$ in $\underline{C}^{n+1}$, it generates a path of approximate $\mathrm{DE}_{\widehat{\alpha}(q)}$ 's in $\widehat{C}^{n+1}$. This part of the price and quantity adjustment process does not correspond to any simplicial algorithm considered before in the literature. Next, a simplicial algorithm will be described that generates the path followed by both the short-term and the long-term price and quantity adjustment process.

To describe the algorithm, let an initial state $v$ in the relative interior of $\underline{C}^{n+1}$ be given. Notice that for any such point it holds that $\widehat{l}_{j}(v)<0, \forall j \in I_{n}$, and $\widehat{L}_{j}(v)>0, \forall j \in$ $I_{n}$. Therefore, there is neither complete supply rationing nor complete demand rationing, and in general no component of $\widehat{z}(v)$ will be equal to zero. The algorithm now proceeds by increasing any component $j$ of $v$ for which $\widehat{z}_{j}(v)>0$ and decreasing any component $j$ of $v$ for which $\widehat{z}_{j}(v)<0$. To formalize this, let $S$ be the set of $n$-dimensional sign vectors given by

$$
S=\left\{s \in \mathbb{R}^{n} \mid s_{j} \in\{-1,0,+1\}, \forall j \in I_{n}\right\}
$$

For any $s \in S$, define the sets $I^{-}(s), I^{0}(s)$, and $I^{+}(s)$ by $I^{-}(s)=\left\{j \in I_{n} \mid s_{j}=-1\right\}$, $I^{0}(s)=\left\{j \in I_{n} \mid s_{j}=0\right\}$, and $I^{+}(s)=\left\{j \in I_{n} \mid s_{j}=+1\right\}$, so $I^{-}(s), I^{0}(s)$, and $I^{+}(s)$ are the set of negative, zero, and positive components of $s$, respectively. Let $i^{-}(s), i^{0}(s)$, and $i^{+}(s)$ denote the number of components in these respective sets. For any $s \in S \backslash\{\underline{0}\}$, define the $i^{0}(s)$-dimensional set $\underline{C}^{n+1}(s)$ by

$$
\underline{C}^{n+1}(s)=\left\{q \in \underline{C}^{n+1} \mid q_{j}=0, \forall j \in I^{-}(s), \text { and } q_{j}=1, \forall j \in I^{+}(s)\right\},
$$




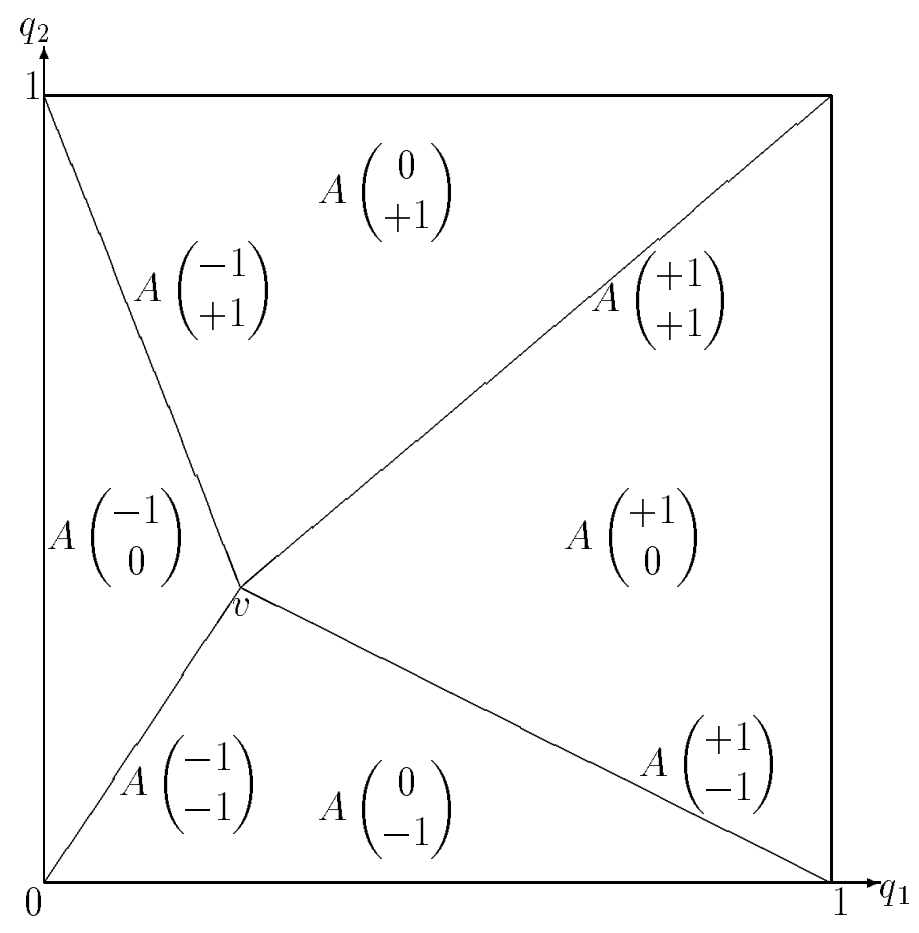

Figure 3: The sets $A(s)$ for $n=2$

and define the $\left(i^{0}(s)+1\right)$-dimensional set $A(s)$ by

$$
A(s)=\operatorname{co}\left(\{v\} \cup \underline{C}^{n+1}(s)\right),
$$

where co denotes the convex hull of a set. Moreover, the set $A(\underline{0})$ is defined by $A(\underline{0})=\widehat{C}^{n+1}$. The sets $A(s), s \neq \underline{0}$, are illustrated for $n=2$ in Figure 3 . Let the first $n$ components of the functions $\widehat{z}$ and $\widehat{Z}$ be denoted by $\underline{\widehat{z}}$ and $\underline{\widehat{Z}}$, respectively. Observe that a point $q \in \widehat{C}^{n+1}$ satisfies the properties of (6) for the function $\widehat{Z}$ if and only if $q \in A(s)$ and $\operatorname{sgn}(\underline{\underline{Z}}(q))=s$ for some $s \in S \backslash\{\underline{0}\}$. Moreover, a point $q \in \widehat{C}^{n+1}$ satisfies property (7) for the function $\widehat{Z}$ if and only if $q \in A(s)$ and $\operatorname{sgn}(\underline{\underline{Z}}(q))=s$ for $s=\underline{0}$.

Now, let $G(\underline{0})$ be a triangulation in $(n+1)$-simplices of the $(n+1)$-dimensional set $A(\underline{0})$ such that for any $s \in S$ the restriction of $G(\underline{0})$ to $A(s)$ induces a triangulation $G(s)$ in $\left(i^{0}(s)+1\right)$-simplices of $A(s)$. Such a triangulation of $\widehat{C}^{n+1}$ is said to be a proper triangulation. A proper triangulation of $\widehat{C}^{n+1}$ with arbitrarily small chosen mesh size exists and can be obtained by adapting the so-called $V$-triangulation on a cube developed in Doup and Talman [5]. For the remainder of this section some proper triangulation of $\widehat{C}^{n+1}$ is assumed to be given.

Let $s \in S$ be a sign vector with $i^{0}(s)=t$, for some $t \in I_{n}^{0}$, and let $\sigma\left(q^{0}, \ldots, q^{t+1}\right)$ be a $(t+1)$-dimensional simplex of $G(s)$. Consider solutions $\left(\bar{\lambda}_{0}, \ldots, \bar{\lambda}_{t+1},\left(\bar{\mu}_{j}\right)_{j \in I^{-}(s) \cup I^{+}(s)}\right) \in$ 
$\mathbb{R}^{n+2}$ of the following system of $(n+1)$ equations:

$$
\sum_{h \in I_{t+1}^{0}} \lambda_{h}\left(\begin{array}{c}
\widehat{z}\left(q^{h}\right) \\
1
\end{array}\right)-\sum_{j \in I^{-}(s) \cup I^{+}(s)} \mu_{j}\left(\begin{array}{c}
s_{j} e(j) \\
0
\end{array}\right)=\left(\begin{array}{c}
\underline{0} \\
1
\end{array}\right),
$$

where $e(j)$ denotes the $j$-th $n$-dimensional unit vector. If $\bar{\lambda}_{h} \geq 0, \forall h \in I_{t+1}^{0}$, and $\bar{\mu}_{j} \geq 0$, $\forall j \in I^{-}(s) \cup I^{+}(s)$, then $\left(\bar{\lambda}_{0}, \ldots, \bar{\lambda}_{t+1},\left(\bar{\mu}_{j}\right)_{j \in I^{-}(s) \cup I^{+}(s)}\right)$ is called an admissible solution to $(9)$.

Notice that if $\sigma\left(q^{0}, \ldots, q^{t+1}\right) \in G(s)$ for some $s \in S$ and $\left(\bar{\lambda}_{0}, \ldots, \bar{\lambda}_{t+1},\left(\bar{\mu}_{j}\right)_{j \in I^{-}(s) \cup I^{+}(s)}\right)$ is an admissible solution to (9), then the point $q$ given by $q=\sum_{h \in I_{t+1}^{0}} \bar{\lambda}_{h} q^{h}$ is an element of $\sigma$ satisfying (6) for $\widehat{Z}$ if $s \neq \underline{0}$ and (7) for $\widehat{Z}$ if $s=\underline{0}$.

An admissible solution $\left(\bar{\lambda}_{0}, \ldots, \bar{\lambda}_{t+1},\left(\bar{\mu}_{j}\right)_{j \in I^{-}(s) \cup I^{+}(s)}\right)$ to $(9)$ such that two or more variables are equal to zero is called a degenerate admissible solution to (9). Following the literature on simplicial algorithms, we make the non-degeneracy assumption that there are no degenerate admissible solutions. Notice that the non-degeneracy assumption is a very weak assumption, since (9) is a system with $n+2$ unknowns and $n+1$ equations, whereas letting two unknowns being equal to zero yields two more equations. Moreover, the number of possible systems, i.e., all simplices in $\cup_{s \in S} G(s)$, is finite. Clearly, if the function $\widehat{z}$ is such that the non-degeneracy assumption is not satisfied, then this assumption will be fulfilled for an arbitrarily small perturbation of $\widehat{z}$. Furthermore, it is possible to circumvent this nondegeneracy assumption completely by using lexicographic pivoting, see Todd [27]. However, lexicographic pivoting implies a much higher mathematical complexity and therefore we simply make the non-degeneracy assumption in this paper. It is important to point out that the non-degeneracy assumption has some interesting economic implications in our model due to the fact that $\widehat{z}$ satisfies the boundary behaviour of Lemma 3.2. It will guarantee that the price and quantity adjustment process will not reach states of the economy with complete supply rationing or complete demand rationing on some market, i.e., if $q$ is generated by the price and quantity adjustment process, then $0<q_{j}<1, \forall j \in I_{n}$. Indeed, the following lemma makes clear that there are no admissible solutions on the boundary of $\widehat{C}^{n+1}$ where $q_{j}=0$ or $q_{j}=1$ for some $j \in I_{n}$ if the non-degeneracy assumption is satisfied.

\section{Lemma 5.2}

Let the economy $\mathcal{E}=\left(\left\{X^{i}, \succeq^{i}, w^{i}\right\}_{i=1}^{m}, \tilde{p}\right)$ satisfy the Assumptions $A 1-A 3$. Let $G$ be $a$ proper triangulation of $\widehat{C}^{n+1}$ and assume that the non-degeneracy assumption is satisfied. Let a sign vector $s \in S$, a simplex $\sigma\left(q^{0}, \ldots, q^{t+1}\right) \in G(s)$ and an admissible solution $\left(\bar{\lambda}_{0}, \ldots, \bar{\lambda}_{t+1},\left(\bar{\mu}_{j}\right)_{j \in I^{-}(s) \cup I^{+}(s)}\right)$ to (9) be given. Let $\bar{q}=\sum_{h \in I_{t+1}^{0}} \bar{\lambda}_{h} q^{h}$. Then $\bar{q}_{j} \in(0,1)$, $\forall j \in I_{n}$.

\section{Proof}

Suppose the lemma is not true and there exists $k \in I_{n}$ such that $\bar{q}_{k}=0$ or $\bar{q}_{k}=1$. First, 
consider the case where $\bar{q}_{k}=0$. Since $v$ is a point of the relative interior of $\underline{C}^{n+1}$ it follows that $k \in I^{-}(s) \cup I^{0}(s)$ and that $\bar{q}$ lies in a facet of $\sigma$, so there is $h^{\prime} \in I_{t+1}^{0}$ with $\bar{\lambda}_{h^{\prime}}=0$. Without loss of generality it can be assumed that $h^{\prime}=t+1$. Therefore, by the non-degeneracy assumption, $\bar{\lambda}_{h}>0, \forall h \in I_{t}^{0}$, and $\bar{\mu}_{j}>0, \forall j \in I^{-}(s) \cup I^{+}(s)$, and hence $q_{k}^{h}=0, \forall h \in I_{t}^{0}$. Notice that $\sum_{h \in I_{t}^{0}} \bar{\lambda}_{h} \widehat{\underline{z}}\left(q^{h}\right)=\sum_{j \in I^{-}(s) \cup I^{+}(s)} \bar{\mu}_{j} s_{j} e(j)$.

Suppose $k \in I^{-}(s)$. Then, using Lemma 3.2, $0 \leq \sum_{h \in I_{t}^{0}} \bar{\lambda}_{h} \widehat{\underline{z}}_{k}\left(q^{h}\right)=-\bar{\mu}_{k}<0$, a contradiction. Suppose $k \in I^{+}(s)$. Then $0=\bar{q}_{k} \geq v_{k}>0$, a contradiction. Consequently, $k \in I^{0}(s)$. Since $k \in I^{0}(s)$ it follows that $\sum_{h \in I_{t}^{0}} \bar{\lambda}_{h} \widehat{\underline{z}}_{k}\left(q^{h}\right)=0$. Since $q_{k}^{h}=0, \forall h \in I_{t}^{0}$, it follows by Lemma 3.2 that $\underline{\underline{z}}_{k}\left(q^{h}\right) \geq 0, \forall h \in I_{t}^{0}$, and therefore $\widehat{\underline{z}}_{k}\left(q^{h}\right)=0, \forall h \in I_{t}^{0}$. Hence, row $k$ of the matrix $M$ given by

$$
M=\left[\left(\begin{array}{c}
\widehat{z}\left(q^{h}\right) \\
1
\end{array}\right)_{h \in I_{t}^{0}},\left(\begin{array}{c}
-s_{j} e(j) \\
0
\end{array}\right)_{j \in I^{-}(s) \cup I^{+}(s)}\right]
$$

is the zero vector and the rank of $M$ is at most $n$. Since the system $M x=\left(\underline{0}^{\top}, 1\right)^{\top}$ has a solution $\left(\bar{\lambda}_{0}, \ldots, \bar{\lambda}_{t},\left(\bar{\mu}_{j}\right)_{j \in I^{-}(s) \cup I^{+}(s)}\right)$ with $\bar{\lambda}_{h}>0, \forall h \in I_{t}^{0}, \bar{\mu}_{j}>0, \forall j \in I^{-}(s) \cup I^{+}(s)$, and since the rank of $M$ is at most $n$, there is a ray of such solutions. Hence there is a solution with $\bar{\lambda}_{h} \geq 0, \forall h \in I_{t}^{0}, \mu_{j} \geq 0, \forall j \in I^{-}(s) \cup I^{+}(s)$, whereas $\bar{\lambda}_{h}=0$ for some $h \in I_{t}^{0}$ or $\bar{\mu}_{j}=0$ for some $j \in I^{-}(s) \cup I^{+}(s)$, a contradiction.

The case where $\bar{q}_{k}=1$ is completely symmetric.

Q.E.D.

For some $s \in S$ and a simplex $\sigma\left(q^{0}, \ldots, q^{t+1}\right) \in G(s)$, let $\left(\bar{\lambda}_{0}, \ldots, \bar{\lambda}_{t+1},\left(\bar{\mu}_{j}\right)_{j \in I^{-}(s) \cup I^{+}(s)}\right)$ be an admissible solution to (9) with $\bar{\lambda}_{h^{\prime}}=0$ for some $h^{\prime} \in I_{t+1}^{0}$. Then the point $\bar{q}=$ $\sum_{h \in I_{t+1}^{0} \backslash\left\{h^{\prime}\right\}} \bar{\lambda}_{h} q^{h}$ is a point satisfying the properties of the adjustment process belonging to the facet $\tau\left(q^{0}, \ldots, q^{h^{\prime}-1}, q^{h^{\prime}+1}, \ldots, q^{t+1}\right)$ of $\sigma$ opposite the vertex $q^{h^{\prime}}$. Such a facet is called an $s$-complete (or complete) facet. More precisely, a facet $\tau\left(q^{0}, \ldots, q^{t}\right)$ of a simplex $\sigma \in G(s)$ is $s$-complete for some sign vector $s \in S$ with $i^{0}(s)=t$ if the system of equations

$$
\sum_{h \in I_{t}^{0}} \lambda_{h}\left(\begin{array}{c}
\widehat{\widehat{z}}\left(q^{h}\right) \\
1
\end{array}\right)-\sum_{j \in I^{-}(s) \cup I^{+}(s)} \mu_{j}\left(\begin{array}{c}
s_{j} e(j) \\
0
\end{array}\right)=\left(\begin{array}{c}
\underline{0} \\
1
\end{array}\right)
$$

has a solution $\left(\bar{\lambda}_{1}, \ldots, \bar{\lambda}_{t},\left(\bar{\mu}_{j}\right)_{j \in I^{-}(s) \cup I^{+}(s)}\right)$ satisfying $\bar{\lambda}_{h} \geq 0, \forall h \in I_{t}^{0}$, and $\bar{\mu}_{j} \geq 0$, $\forall j \in I^{-}(s) \cup I^{+}(s)$, called an admissible solution to (10). Notice that the non-degeneracy assumption implies $\bar{\lambda}_{h}>0, \forall h \in I_{t}^{0}$, and $\bar{\mu}_{j}>0, \forall j \in I^{-}(s) \cup I^{+}(s)$. Two facets $\tau$ and $\bar{\tau}$ are said to be adjacent complete facets if

(i) $\tau \neq \bar{\tau}$ and for some $s$, both $\tau$ and $\bar{\tau}$ are $s$-complete facets of the same simplex $\sigma \in G(s)$, or

(ii) $\tau \neq \bar{\tau}$, for some $s \neq \underline{0}, \tau$ is an $s$-complete facet of $\bar{\tau}$ and there exists some $j \in$ $I^{-}(s) \cup I^{+}(s)$ such that $\bar{\tau}$ is an $\bar{s}$-complete simplex in $G(s)$ with $\bar{s}_{j}=0$ and $\bar{s}_{k}=s_{k}$ for all 
$k \neq j$, or

(iii) $\tau=\bar{\tau}$ and for some $s \neq \underline{0}, j \in I^{-}(s) \cup I^{+}(s), k \in I^{0}(s)$ we have that $\tau$ is both an $s$-complete facet of a simplex $\sigma \in G(s)$ and $\bar{\tau}$ is an $\bar{s}$-complete facet of a simplex $\bar{\sigma} \in G(\bar{s})$ with $\bar{s}_{j}=0, \bar{s}_{k} \in\{-1,+1\}$ and $\bar{s}_{h}=s_{h}$ for all $h \neq j, k$.

Consider the simplex $\{v\}$. This 0 -simplex can only be an $s$-complete facet for some $s \in S$ if $i^{0}(s)=0$. It follows easily that $\{v\}$ is an $s$-complete facet of the unique simplex $\sigma\left(v, q^{1}\right) \in G(s)$ containing $\{v\}$ as a facet, where $s=\operatorname{sgn}(\underline{\underline{z}}(v))$ and the admissible solution to the system in (10) corresponding to $s$ and $\{v\}$ is given by $\lambda_{0}=1$ and $\mu_{j}=\left|\underline{z}_{j}(v)\right|$, $\forall j \in I_{n}$. The admissible solution corresponding to the system in (9) for the simplex $\sigma\left(v, q^{1}\right)$ is given by $\lambda_{0}=1, \lambda_{1}=0$ and $\mu_{j}=\left|\underline{\underline{z}}_{j}(v)\right|, \forall j \in I_{n}$. Notice that the non-degeneracy assumption implies $\mu_{j}>0, \forall j \in I_{n}$. Hence, it follows immediately that there is no other sign vector $s \in S$ for which $\{v\}$ is $s$-complete.

The following lemma shows that there is exactly one adjacent complete facet to $\{v\}$. Moreover, if $\tau$ is an $s$-complete facet belonging to $\bar{C}^{n+1}$, (hence $\tau$ is a $\underline{0}$-complete facet), then there is also exactly one adjacent complete facet to $\tau$. Finally, if $\tau$ is an $s$-complete facet for some $s \in S$, and $\tau$ is not equal to $\{v\}$ and does not belong to $\bar{C}^{n+1}$, then there are exactly two adjacent complete facets to $\tau$.

\section{Lemma $\mathbf{5 . 3}$}

Let the economy $\mathcal{E}=\left(\left\{X^{i}, \succeq^{i}, w^{i}\right\}_{i=1}^{m}, \tilde{p}\right)$ satisfy the Assumptions A1-A3. Let $G$ be a proper triangulation of $\widehat{C}^{n+1}$ and assume that the non-degeneracy assumption is satisfied. Let a sign vector $s \in S$ and an s-complete facet $\tau\left(q^{0}, \ldots, q^{t}\right)$ of a simplex in $G(s)$ be given. If $\tau=\{v\}$ or if $\tau \subset \bar{C}^{n+1}$, then there is exactly one adjacent complete simplex to $\tau$. Otherwise, there are exactly two adjacent complete simplices to $\tau$.

\section{Proof}

Let $\tau=\{v\}$. Then $\tau$ is $s$-complete for a uniquely determined sign vector $s \in S$. Since $\tau$ lies in the relative boundary of $A(s)$ there is a unique simplex $\sigma\left(v, q^{1}\right) \in G(s)$ having $\tau$ as a facet. Make a linear programming pivot step with the vector $\left(\underline{z}\left(q^{1}\right)^{\top}, 1\right)^{\top}$ in the system (10) corresponding to $s$ and $\tau$. By the non-degeneracy assumption exactly one of the variables $\lambda_{0}, \mu_{j}, j \in I_{n}$, becomes zero. If $\lambda_{0}$ becomes zero, then $\left\{q^{1}\right\}$ is an $s$-complete facet of a simplex in $G(s)$. If, say, $\mu_{j^{\prime}}=0$, then $\sigma\left(v, q^{1}\right)$ is an $\bar{s}$-complete facet of a uniquely determined simplex in $G(\bar{s})$, where $\bar{s}_{j^{\prime}}=0$ and $\bar{s}_{j}=s_{j}, \forall j \in I_{n} \backslash\left\{j^{\prime}\right\}$. This yields exactly one adjacent complete simplex to $\tau$. It is clear that there cannot be any other.

Let $\tau\left(q^{0}, \ldots, q^{t}\right)$ be an $s$-complete facet in $\bar{C}^{n+1}$. Then, $\tau$ is $\underline{0}$-complete and $t=n$. Since $\tau$ lies in the relative boundary of $A(\underline{0})$ there is exactly one simplex, say $\sigma\left(q^{0}, \ldots, q^{n+1}\right)$, in $G(\underline{0})$ having $\tau$ as a facet. Make a linear programming pivot step with the vector $\left(\underline{\underline{z}}\left(q^{n+1}\right)^{\top}, 1\right)^{\top}$ in the system (10) corresponding to $s=\underline{0}$ and $\tau$. By the non-degeneracy assumption exactly one of the variables $\lambda_{h}, h \in I_{n}^{0}$, becomes zero, say $\lambda_{h^{\prime}}$. Therefore, the 
facet of $\sigma$ opposite the vertex $\left\{q^{h^{\prime}}\right\}$ is a $\underline{0}$-complete facet of a simplex in $G(\underline{0})$. This yields exactly one adjacent complete simplex to $\tau$. It is clear that there cannot be any other adjacent complete simplex.

Let $\tau\left(q^{0}, \ldots, q^{t}\right)$ be an $s$-complete facet such that neither $\tau=\{v\}$ nor $\tau$ is a subset of $\bar{C}^{n+1}$. There are two possibilities: either $\tau$ lies in the relative boundary of $A(s)$ or $\tau$ lies in the relative interior of $A(s)$. Suppose $\tau$ lies in the relative boundary of $A(s)$. Then there is a unique simplex $\sigma\left(q^{0}, \ldots, q^{t+1}\right)$ in $G(s)$ containing $\tau$ as a facet. Make a linear programming pivot step with the vector $\left(\underline{\underline{z}}\left(q^{t+1}\right)^{\top}, 1\right)^{\top}$ in the system (10) corresponding to $s$ and $\tau$. By the non-degeneracy assumption exactly one of the variables $\lambda_{h}, h \in I_{t}^{0}, \mu_{j}$, $j \in I^{-}(s) \cup I^{+}(s)$, becomes zero. If $\lambda_{h^{\prime}}$ becomes zero, then the facet of $\sigma$ opposite $q^{h^{\prime}}$ is an $s$-complete facet of a simplex in $G(s)$. If $\mu_{j^{\prime}}=0$, then $\sigma$ is an $\bar{s}$-complete facet of a uniquely determined simplex in $G(\bar{s})$, where $\bar{s}_{j^{\prime}}=0$ and $\bar{s}_{j}=s_{j}, \forall j \in I_{n} \backslash\left\{j^{\prime}\right\}$. This yields exactly one adjacent complete simplex to $\tau$. Since $\tau$ lies in the relative boundary of $A(s)$, but $\tau$ is neither a subset of $\bar{C}^{n+1}$ nor of the boundary of $A(s)$ where $q_{j}=0$ or $q_{j}=1$ for some $j \in I_{n}$ by Lemma 5.2, it holds that $\tau$ is a simplex of $G(\widehat{s})$ for a unique sign vector $\widehat{s} \in S$ with $i^{0}(\widehat{s})=t-1$. Let $j^{\prime} \in I^{0}(s)$ be the unique component such that $\widehat{s}_{j^{\prime}} \neq 0$. Make a linear programming pivot step with the vector $\left(s_{j^{\prime}} e\left(j^{\prime}\right)^{\top}, 0\right)^{\top}$ in the system (10) corresponding to $s$ and $\tau$. By the non-degeneracy assumption exactly one of the variables $\lambda_{h}, h \in I_{t}^{0}, \mu_{j}$, $j \in I^{-}(s) \cup I^{+}(s)$, becomes zero. If $\lambda_{h^{\prime}}$ becomes zero, then the facet of $\tau$ opposite $q^{h^{\prime}}$ is an $\widehat{s}$-complete facet of $\tau$. If $\mu_{\hat{\jmath}}=0$, then $\tau$ is an $\widetilde{s}$-complete facet of a uniquely determined simplex $\sigma\left(q^{0}, \ldots, q^{t}, \widetilde{q}^{t+1}\right)$ in $G(\widetilde{s})$, where $\widetilde{s}_{\hat{\jmath}}=0$ and $\widetilde{s}_{j}=\widehat{s}_{j}, \forall j \in I_{n} \backslash\{\hat{\jmath}\}$. In this case $\tau$ is both an $s$-complete facet of a simplex in $G(s)$ and an $\widetilde{s}$-complete facet of a simplex in $G(\widetilde{s})$, with $s \neq \widetilde{s}$, and is therefore adjacent complete to itself. It is clear that there cannot be any other.

Consider the case with $\tau$ lying in the relative interior of $A(s)$. Then there are exactly two simplices, say $\sigma\left(q^{0}, \ldots, q^{t+1}\right)$ and $\sigma\left(q^{0}, \ldots, q^{t}, \bar{q}^{t+1}\right)$, containing $\tau$ as a facet. Make a linear programming pivot step with the vector $\left(\underline{\underline{z}}\left(q^{t+1}\right)^{\top}, 1\right)^{\top}$ and with the vector $\left(\underline{\hat{z}}\left(\bar{q}^{t+1}\right)^{\top}, 1\right)^{\top}$ respectively in the system (10) corresponding to $s$ and $\tau$. Using the same arguments as before, this yields exactly two adjacent complete simplices to $\tau$. It is clear that there cannot be any other.

Q.E.D.

\section{Lemma 5.4}

Let the economy $\mathcal{E}=\left(\left\{X^{i}, \succeq^{i}, w^{i}\right\}_{i=1}^{m}, \tilde{p}\right)$ satisfy the Assumptions A1-A3. Let $G$ be $a$ proper triangulation of $\widehat{C}^{n+1}$ and assume that the non-degeneracy assumption is satisfied. Then there exists a unique finite sequence of different pairs of sign vectors and simplices $\left(s^{1}, \tau^{1}\right), \ldots,\left(s^{M}, \tau^{M}\right)$ such that $\tau^{1}=\{v\}, \tau^{M} \subset \bar{C}^{n+1}, \tau^{k}$ is an $s^{k}$-complete facet and any two successive simplices in the sequence are adjacent complete simplices. 


\section{Proof}

Let $\tau^{1}=\{v\}$, which is $\operatorname{sgn}(\underline{\underline{z}}(v))$-complete. Let $\tau^{2}$, with corresponding sign vector $s^{2}$, be the unique adjacent complete simplex to $\tau^{1}$, which exists according to Lemma 5.3. Now, for $k \in \mathbb{N} \backslash\{1\}$, it holds that either $\tau^{k} \subset \bar{C}^{n+1}$ and there exists no adjacent complete simplex other than $\tau^{k-1}$ by Lemma 5.3, or there exists by Lemma 5.3 a unique simplex $\tau^{k+1}$, with corresponding sign vector $s^{k+1}$, that is not equal to $\tau^{k-1}$. Proceeding this way, there exists a finite number $M$ such that either $\tau^{K} \subset \bar{C}^{n+1}$ or, by the finiteness of the number of pairs $(s, \tau)$ such that $\tau$ is an $s$-complete facet of a simplex in $G(s)$, the pair $\left(s^{M}, \tau^{M}\right)$ has been generated before. However, from the door-in-door-out principle of Lemke and Howson [19] it follows that each pair $(s, \tau)$, representing an $s$-complete facet $\tau$ of a simplex in $G(s)$ for some $s \in S$, can be generated at most once. Hence, $\tau^{M} \subset \bar{C}^{n+1}$.

Q.E.D.

The algorithm generating the sequence of adjacent complete simplices as described in Lemma 5.4 is given below. In the description of the algorithm, $\tau^{k}=\operatorname{co}\left(\left\{q^{h} \mid h \in I_{t}^{0}\right\}\right)$ will denote an $s^{k}$-complete facet currently generated by the procedure, where $s^{k}$ with $i^{0}\left(s^{k}\right)=t$ is a sign vector generated by the procedure and induces the set $A\left(s^{k}\right)$ and the triangulation $G\left(s^{k}\right)$ of $A\left(s^{k}\right)$ in which the procedure generates simplices. The algorithm operates as follows.

\section{Algorithm}

Step 0. Set $t=0$ and $k=1$. Set $\tau^{k}=\{v\}, s^{k}=\operatorname{sgn}(\underline{\widehat{z}}(v))$ and let $q^{t+1}$ be the unique point in $A\left(s^{k}\right)$ such that $\sigma^{k}\left(v, q^{t+1}\right)$ is a $(t+1)$-simplex of $G\left(s^{k}\right)$. Go to Step 1 .

Step 1. Let $\sigma$ be equal to the convex hull of $\tau^{k} \cup\left\{q^{t+1}\right\}$. Make a linear programming pivot step with the vector $\left(\underline{\underline{z}}\left(q^{t+1}\right)^{\top}, 1\right)^{\top}$ in the system (10) corresponding to $s^{k}$ and $\tau^{k}$. Then exactly one of the variables $\lambda_{h}, h \in I_{t}^{0}, \mu_{j}, j \in I^{-}\left(s^{k}\right) \cup I^{+}\left(s^{k}\right)$, becomes equal to zero. If for some $h^{\prime} \in I_{t}^{0}, \lambda_{h^{\prime}}=0$, then set $s^{k+1}=s^{k}$ and go to Step 2. If for some $j^{\prime} \in I^{-}\left(s^{k}\right) \cup I^{+}\left(s^{k}\right), \mu_{j^{\prime}}=0$, then set $\bar{s}=s^{k}$ and go to Step 3 .

Step 2. Increase the value of $k$ by 1 and let $\tau^{k}$ be the facet of $\sigma$ opposite $q^{h^{\prime}}$. If $\tau^{k} \subset \bar{C}^{n+1}$, then the algorithm terminates. If $\tau^{k} \in G(\bar{s})$ for some $\bar{s} \in S$, then go to Step 4 . Otherwise, there is exactly one $(t+1)$-simplex $\bar{\sigma} \in G\left(s^{k}\right)$ such that $\bar{\sigma} \neq \sigma$ and $\tau^{k}$ is a facet of $\bar{\sigma}$. Go to Step 1 with $q^{t+1}$ as the unique vertex of $\bar{\sigma}$ opposite $\tau^{k}$.

Step 3. Define $s^{k+1}$ by $s_{j^{\prime}}^{k+1}=0$ and $s_{j}^{k+1}=\bar{s}_{j}, \forall j \in I_{n} \backslash\left\{j^{\prime}\right\}$. There is a unique simplex $\bar{\sigma} \in G\left(s^{k+1}\right)$ having $\sigma$ as a facet. Increase the values of $k$ and $t$ by 1 and go to Step 1 with $q^{t+1}$ as the unique vertex of $\bar{\sigma}$ opposite $\sigma$ and $\tau^{k}=\sigma$.

Step 4. Let $\sigma$ be equal to $\tau^{k}$. Make a linear programming pivot step with the vector $\left(\bar{s}_{j} e(j)^{\top}, 0\right)^{\top}$ in the system (10) corresponding to $s^{k}$ and $\tau^{k}$, where $j \in I_{n}$ is such 
that $s_{j}^{k}=0$ and $\bar{s}_{j} \neq 0$. Then exactly one of the variables $\lambda_{h}, h \in I_{t}^{0}, \mu_{j}, j \in$ $I^{-}\left(s^{k}\right) \cup I^{+}\left(s^{k}\right)$, becomes equal to zero. If for some $h^{\prime} \in I_{t}^{0}, \lambda_{h^{\prime}}=0$, then set $s^{k+1}=\bar{s}$, decrease the value of $t$ by 1 and go to Step 2. If for some $j^{\prime} \in I^{-}\left(s^{k}\right) \cup I^{+}\left(s^{k}\right), \mu_{j^{\prime}}=0$, then decrease the value of $t$ by 1 and go to Step 3 .

Let the assumptions of Lemma 5.4 be satisfied and let $\left(s^{1}, \tau^{1}\right), \ldots,\left(s^{M}, \tau^{M}\right)$ be all different pairs of sign vectors and simplices successively generated by the algorithm. For every pair $\left(s^{k}, \tau^{k}\right), k \in I_{M}$, it holds that $\tau^{k}$ is an $s^{k}$-complete facet of an $\left(i^{0}\left(s^{k}\right)+1\right)$-simplex of $G\left(s^{k}\right)$. For every $k \in I_{M}$, define $t^{k}=i^{0}\left(s^{k}\right)$, let $\tau^{k}=\tau^{k}\left(q^{0}, \ldots, q^{t^{k}}\right)$ and define $\bar{q}^{k} \in \tau^{k}$ by

$$
\bar{q}^{k}=\sum_{h \in I_{t^{k}}^{0}} \bar{\lambda}_{h} q^{h},
$$

with $\bar{\lambda}_{h}$ following from the admissible solution to (10) corresponding to $s^{k}$ and $\tau^{k}$. For $t \in \mathbb{R}$, define $\lfloor t\rfloor$ as the smallest integer which is less than or equal to $t$. Finally, define the piecewise linear, continuous function $\pi:[0,1] \rightarrow \widehat{C}^{n+1}$ by

$$
\begin{aligned}
\pi(t)= & (1-(M-1) t+\lfloor(M-1) t\rfloor) \bar{q}^{1+\lfloor(M-1) t\rfloor} \\
& +((M-1) t-\lfloor(M-1) t\rfloor) \bar{q}^{2+\lfloor(M-1) t\rfloor}, \forall t \in[0,1), \\
\pi(1)= & \bar{q}^{M} .
\end{aligned}
$$

Clearly, $\pi$ generates a piecewise linear path in $\widehat{C}^{n+1}$ connecting the point $q^{1}=\{v\}$ with a point $q^{M} \in \bar{C}^{n+1}$. Furthermore, every point $q \in \pi([0,1]) \cap \underline{C}^{n+1}$ satisfies (6) for the piecewise linear approximation $\widehat{Z}$, and every point $q \in \pi([0,1]) \cap\left(\widehat{C}^{n+1} \backslash \underline{C}^{n+1}\right)$ satisfies (7) for $\widehat{Z}$, as is proved in the following theorem.

\section{Theorem 5.5}

Let the economy $\mathcal{E}=\left(\left\{X^{i}, \succeq^{i}, w^{i}\right\}_{i=1}^{m}, \widetilde{p}\right)$ satisfy the Assumptions A1-A3. Let $G$ be a proper triangulation of $\widehat{C}^{n+1}$ and assume that the non-degeneracy assumption is satisfied. Then, for every $q \in \pi([0,1]) \cap \underline{C}^{n+1}$ there exists a real number $\gamma \in[0,1]$ such that, for every $j \in I_{n}$,

$$
\begin{array}{ll}
q_{j}=\gamma v_{j} & \text { if } \widehat{Z}_{j}(q)<0, \\
\gamma q_{j} \leq q_{j} \leq 1-\gamma\left(1-v_{j}\right) & \text { if } \widehat{Z}_{j}(q)=0, \\
q_{j}=1-\gamma\left(1-v_{j}\right) & \text { if } \widehat{Z}_{j}(q)>0 .
\end{array}
$$

Furthermore, for all $q \in \pi([0,1]) \cap\left(\widehat{C}^{n+1} \backslash \underline{C}^{n+1}\right)$ it holds that $\widehat{Z}(q)=0$.

\section{Proof}

Consider the pairs $\left(s^{k}, \tau^{k}\right)$ and $\left(s^{k+1}, \tau^{k+1}\right)$ for some $k \in I_{M-1}$. Let $\sigma^{k}=\operatorname{co}\left(\tau^{k} \cup \tau^{k+1}\right)$ and let $\bar{s}^{k}$ be such that $I^{-}\left(\bar{s}^{k}\right)=I^{-}\left(s^{k}\right) \cup I^{-}\left(s^{k+1}\right), I^{0}\left(\bar{s}^{k}\right)=I^{0}\left(s^{k}\right) \cap I^{0}\left(s^{k+1}\right)$ and 
$I^{+}\left(\bar{s}^{k}\right)=I^{+}\left(s^{k}\right) \cup I^{+}\left(s^{k+1}\right)$. For $\bar{q}^{k}$ and $\bar{q}^{k+1}$, induced by the admissible solutions to (10) corresponding to $\left(s^{k}, \tau^{k}\right)$ respectively $\left(s^{k+1}, \tau^{k+1}\right)$, it holds that they are also induced by admissible solutions $y^{k}, y^{k+1} \in \mathbb{R}^{n+2}$ to $(9)$ corresponding to $\left(\bar{s}^{k}, \sigma^{k}\right)$. When $y^{k}, y^{k+1}$ are admissible solutions, then so is $\lambda y^{k}+(1-\lambda) y^{k+1}, \forall \lambda \in[0,1]$. Then, since $\sigma^{k} \in G\left(\bar{s}^{k}\right)$ for all $k \in I_{M-1}$, it follows that for every $q \in \pi([0,1]) \cap \underline{C}^{n+1}$, and thus $q \in G(s)$ for some $s \neq \underline{0},(6)$ is satisfied for $\widehat{Z}$, whereas for every $q \in \pi([0,1]) \cap\left(\widehat{C}^{n+1} \backslash \underline{C}^{n+1}\right)$, and thus $q \in G(\underline{0}),(7)$ is satisfied for $\widehat{Z}$.

Q.E.D.

\section{Accuracy Analysis}

In Section 5 we have proved, for an arbitrary piecewise linear approximation $\widehat{Z}$ of $\widehat{z}$, the existence of a piecewise linear path of points in $\widehat{C}^{n+1}$ that connects the starting point $\{v\}$ with a zero point of $\widehat{Z}$ in $\bar{C}^{n+1}$. For every point along the path that belongs to $\underline{C}^{n+1},(6)$ holds for the piecewise linear approximation, whereas for every point along the path that belongs to $\widehat{C}^{n+1} \backslash \underline{C}^{n+1},(7)$ holds for the approximation.

In this section it will be shown that this path follows the price and quantity adjustment process as described in Section 4 arbitrarily close by taking the mesh-size of the triangulation small enough. To this end, observe that the properties, as given by (6), of points

$q \in \underline{C}^{n+1} \backslash\{v\}$ that are reached by the process are equivalent to

$$
\begin{array}{ll}
\widehat{z}_{j}(q) \leq 0 & \text { if } q_{j}=\gamma v_{j}, \\
\widehat{z}_{j}(q)=0 & \text { if } \gamma v_{j}<q_{j}<1-\gamma\left(1-v_{j}\right), \\
\widehat{z}_{j}(q) \geq 0 & \text { if } q_{j}=1-\gamma\left(1-v_{j}\right) .
\end{array}
$$

For $q=v$ the properties in (6) are trivially satisfied by taking $\gamma=1$, both for $\widehat{z}$ and for $\widehat{Z}$. The following result shows that the other points related to the adjustment process for $\widehat{Z}$ approximately satisfy the properties $(6)$ and $(7)$ for $\widehat{z}$.

\section{Theorem 6.1}

Let the economy $\mathcal{E}=\left(\left\{X^{i}, \succeq^{i}, w^{i}\right\}_{i=1}^{m}, \tilde{p}\right)$ satisfy the Assumptions A1-A3. Then, for every $\varepsilon>0$ there exists $\delta>0$ such that for every proper triangulation $G$ of $\widehat{C}^{n+1}$ satisfying $\operatorname{mesh}(G)<\delta$ and for which the non-degeneracy assumption is satisfied, it holds that

$$
\begin{aligned}
& \text { for every } q \in \pi((0,1]) \cap \underline{C}^{n+1}, \text { for every } j \in I_{n}, \\
& \qquad \begin{array}{ll}
\widehat{z}_{j}(q)<\varepsilon & \text { if } q_{j}=\gamma v_{j}, \\
-\varepsilon<\widehat{z}_{j}(q)<\varepsilon & \text { if } \gamma v_{j}<q_{j}<1-\gamma\left(1-v_{j}\right), \\
\widehat{z}_{j}(q)>-\varepsilon & \text { if } q_{j}=1-\gamma\left(1-v_{j}\right) .
\end{array}
\end{aligned}
$$

(ii) for every $q \in \pi([0,1]) \cap\left(\widehat{C}^{n+1} \backslash \underline{C}^{n+1}\right)$,

$$
\|\widehat{z}(q)\|_{\infty}<\varepsilon .
$$




\section{Proof}

Given $\varepsilon>0$, define $\varepsilon^{\prime}=(1-\bar{\beta})\left(\sum_{j \in I_{n}} \widetilde{p}_{j}\right)^{-1} \varepsilon$ and let $\bar{\varepsilon}=\min \left\{\varepsilon, \varepsilon^{\prime}\right\}$. Since $\widehat{C}^{n+1}$ is compact and $\widehat{z}$ is continuous, there exists $\delta>0$ such that $q^{1}, q^{2} \in \widehat{C}^{n+1}$ and $\left\|q^{1}-q^{2}\right\|_{\infty}<\delta$, implies $\left\|\widehat{z}\left(q^{1}\right)-\widehat{z}\left(q^{2}\right)\right\|_{\infty}<\bar{\varepsilon}$. Let $G$ be a proper triangulation of $\widehat{C}^{n+1} \operatorname{satisfying} \operatorname{mesh}(G)<\delta$ and being such that the non-degeneracy assumption is satisfied. Furthermore, for all $q \in \widehat{C}^{n+1}$ there exists $\lambda \in \mathbb{R}_{+}^{n+1}$ such that $\sum_{j \in I_{n+1}} \lambda_{j}=1$ and $q=\sum_{j \in I_{n+1}} \lambda_{j} q^{j}$, with $q^{j}$ the vertices of an $n$-simplex $\sigma\left(q^{1}, \ldots, q^{n+1}\right)$ of $G$ containing $q$. Consequently, for all $q \in \widehat{C}^{n+1}$,

$$
\|\widehat{Z}(q)-\widehat{z}(q)\|_{\infty}=\left\|\sum_{j \in I_{n+1}} \lambda_{j}\left(\widehat{z}\left(q^{j}\right)-\widehat{z}(q)\right)\right\|_{\infty}<\bar{\varepsilon}
$$

and therefore, for all $j \in I_{n+1}$,

$$
\widehat{Z}_{j}(q)-\bar{\varepsilon}<\widehat{z}_{j}(q)<\widehat{Z}_{j}(q)+\bar{\varepsilon} .
$$

Now, for every $q \in \pi((0,1]) \cap \underline{C}^{n+1}$ there exists $\mu_{j} \in \mathbb{R}_{+}, \forall j \in I_{n}$, and $\gamma \in[0,1]$, such that for every $j \in I_{n}$,

$$
\begin{array}{ll}
\widehat{Z}_{j}(q)=-\mu_{j} & \text { if } q_{j}=\gamma v_{j}, \\
\widehat{Z}_{j}(q)=0 & \text { if } \gamma v_{j}<q_{j}<1-\gamma\left(1-v_{j}\right), \\
\widehat{Z}_{j}(q)=\mu_{j} & \text { if } q_{j}=1-\gamma\left(1-v_{j}\right) .
\end{array}
$$

Combining this with (11) yields part (i) of the theorem. For $q \in \pi([0,1]) \cap\left(\widehat{C}^{n+1} \backslash \underline{C}^{n+1}\right)$ it holds that $\widehat{Z}_{j}(q)=0, \forall j \in I_{n}$, and thus, using (11), $\left|\widehat{z}_{j}(q)\right|<\bar{\varepsilon}$. Consequently, $\left|\sum_{j \in I_{n}} \widehat{p}_{j}(q) \widehat{z}_{j}(q)\right| \leq \sum_{j \in I_{n}}\left|\widehat{p}_{j}(q) \widehat{z}_{j}(q)\right|<(1-\bar{\beta})^{-1} \sum_{j \in I_{n}} \widetilde{p}_{j} \bar{\varepsilon} \leq \varepsilon$. But then, since $\widehat{p}(q)^{\top} \widehat{z}(q)=0$ by Lemma 3.1, we have that $\left|\widehat{z}_{n+1}(q)\right|=\left|-\sum_{j \in I_{n}} \widehat{p}_{j}(q) \widehat{z}_{j}(q)\right|<\varepsilon$. Q.E.D.

Notice that Theorem 6.1 implies that for every $\varepsilon>0$ there exists $\delta>0$ such that for every proper triangulation $G$ of $\widehat{C}^{n+1}$ with $\operatorname{mesh}(G)<\delta$ and for which the non-degeneracy assumption is satisfied it holds that every point $q$ in $\widehat{C}^{n+1} \backslash \underline{C}^{n+1}$ generated by the algorithm is an $\varepsilon$ - $\mathrm{DE}_{\widehat{\alpha}(q)}$ and that every point $q$ in $\bar{C}^{n+1}$ generated by the algorithm is an $\varepsilon$-WE. This follows easily once it is shown that demand rationing on the market of commodity $j \in I_{n}$ cannot be binding for such a point $q$ if $q_{j} \leq \frac{2}{3}$. Without loss of generality assume that $\varepsilon<\min _{i \in I_{m}, j \in I_{n}}\left(\frac{\tilde{p} \cdot w}{\widetilde{p}_{j}}-w_{j}+w_{j}^{i}\right)$. Then, for every $i \in I_{m}$, for every $j \in I_{n}$, it follows that

$$
\widehat{d}_{j}^{i}(q)-w_{j}^{i} \leq \widehat{z}_{j}(q)+w_{j}-w_{j}^{i}<\varepsilon+w_{j}-w_{j}^{i}<\frac{\widetilde{p} \cdot w}{\widetilde{p}_{j}}=\widehat{L}_{j}(q) \text { if } q_{j} \leq \frac{2}{3} .
$$

The next result shows that the path of the approximate adjustment process gets arbitrarily close to the set $Q_{v}$. This does not exclude that the set $Q_{v}$ contains points which are never approximated by the adjustment process related to $\widehat{Z}$. For instance, in case the set $Q_{v}$ displays a bifurcation, it is possible that the approximate adjustment process converges to one of the branches. In case the adjustment process related to $\widehat{z}$ is convergent, also the 
converse follows, i.e., every point of $Q_{v}$ gets arbitrarily close to the path of the approximate adjustment process. It follows that if the adjustment process related to $\widehat{z}$ is convergent, then any sequence of paths related to the approximate adjustment process with mesh size going to zero converges to $Q_{v}$. For a non-empty compact set $S$ of $\mathbb{R}^{k}$ define the distance function $g_{S}: \mathbb{R}^{k} \rightarrow \mathbb{R}$ by

$$
g_{S}(\bar{s})=\min _{s \in S}\|s-\bar{s}\|_{2}, \forall \bar{s} \in \mathbb{R}^{k} .
$$

Notice that both the sets $Q_{v}$ and $\pi([0,1])$ are compact.

\section{Theorem 6.2}

Let the economy $\mathcal{E}=\left(\left\{X^{i}, \succeq^{i}, w^{i}\right\}_{i=1}^{m}, \tilde{p}\right)$ satisfy the Assumptions A1-A3. Then, for every $\varepsilon>0$ there exists $\delta>0$ such that for every proper triangulation $G$ of $\widehat{C}^{n+1}$ satisfying $\operatorname{mesh}(G)<\delta$ and for which the non-degeneracy assumption is satisfied, it holds that, for every $t \in[0,1], g_{Q_{v}}(\pi(t))<\varepsilon$. If the price and quantity adjustment process is convergent then, for every $\varepsilon>0$ there exists $\delta>0$ such that for every proper triangulation $G$ of $\widehat{C}^{n+1}$ satisfying mesh $(G)<\delta$ and for which the non-degeneracy assumption is satisfied, it holds that, for every $q \in Q_{v}, g_{\pi([0,1])}(q)<\varepsilon$.

\section{Proof}

Suppose the first part of the theorem is not true. Then there exists $\varepsilon>0$ such that for every $r \in \mathbb{N}$ there exists a proper triangulation $G^{r}$ of $\widehat{C}^{n+1} \operatorname{satisfying} \operatorname{mesh}\left(G^{r}\right)<\frac{1}{r}$, for which the non-degeneracy assumption is satisfied, and there exists $t^{r} \in[0,1]$ such that $g_{Q_{v}}\left(\pi^{r}\left(t^{r}\right)\right) \geq \varepsilon$. By Hildenbrand [12], Proposition 1, page 16 , the sequence $\left\{\pi^{r}([0,1])\right\}_{r \in \mathbb{N}}$ has a convergent subsequence which we also denote by $\left\{\pi^{r}([0,1])\right\}_{r \in \mathbb{N}}$. Using the continuity of $\widehat{z}$ and Theorem 6.1 it follows easily that the closed limit of this sequence is a subset of $Q$. By Mas-Colell [20], Theorem A.5.1.(ii), page 10, the closed limit is connected since every $\pi^{r}([0,1])$ is connected. Therefore, the closed limit is a subset of $Q_{v}$. Consider any accumulation point of the sequence $\left\{\pi^{r}\left(t^{r}\right)\right\}_{r \in \mathbb{N}}$, say $\bar{q}$, so $\bar{q} \in Q_{v}$. So,

$$
0=g_{Q_{v}}(\bar{q}) \geq \inf \left\{g_{Q_{v}}\left(\pi^{r}\left(t^{r}\right)\right) \mid r \in \mathbb{N}\right\} \geq \varepsilon,
$$

a contradiction.

Suppose the second part of the theorem is not true. Then there exists $\varepsilon>0$ such that for every $r \in \mathbb{N}$ there exists a proper triangulation $G^{r}$ of $\widehat{C}^{n+1}$ satisfying $\operatorname{mesh}(G)<\delta$ for which the non-degeneracy assumption is satisfied, and there exists $q^{r} \in Q_{v}$ such that $g_{\pi^{r}([0,1])}\left(q^{r}\right) \geq \varepsilon$. Without loss of generality, the sequence $\left\{q^{r}\right\}_{r \in \mathbb{N}}$ is convergent, say to the element $\bar{q}$. Also without loss of generality, by Hildenbrand [12], Proposition 1, page 16, the sequence $\left\{\pi^{r}([0,1])\right\}_{r \in \mathbb{N}}$ is convergent, say to the set $\Pi$. By the first part of the theorem it follows that $\Pi \subset Q_{v}$. Clearly, $\bar{q} \notin \Pi$. Since the adjustment process $Q_{v}$ is convergent there 
exists a homeomorphism $f:[0,1] \rightarrow Q_{v}$, where $f(0)=v$ and $f(1)=q^{*}$ with $q^{*}$ inducing a Walrasian equilibrium of $\mathcal{E}$ and $q_{n+1}^{*}=\bar{\beta}$. Using that $Q_{v}$ is an arc and that there exists a neighbourhood $N$ of $q^{*}$ such that $q \in N$ and $\widehat{z}(q)=\underline{0}$ implies that $q$ induces a Walrasian equilibrium of $\mathcal{E}$, it follows that there is no $q \in Q_{v}$ such that $q \neq q^{*}$ and $q_{n+1}=\bar{\beta}$. Hence, $\pi^{r}(0) \rightarrow f(0)=v$ and $\pi^{r}(1) \rightarrow f(1)=q^{*}$. Moreover, there is $\bar{t} \in(0,1)$ such that $\bar{q}=f(\bar{t})$. By Mas-Colell [20], Theorem A.5.1.(ii), page 10, $\Pi$ is connected. However, $\Pi \subset f([0,1] \backslash\{\bar{t}\}), f(0) \in \Pi, f(1) \in \Pi$ and $f$ is a homeomorphism, so $\Pi$ is not connected, a contradiction.

Q.E.D.

\section{References}

[1] J.-P. Bénassy, Nonclearing markets: microeconomic concepts and macroeconomic applications, Journal of Economic Literature 31 (1993) 732-761.

[2] V. Böhm, Recurrence in Keynesian macroeconomic models, in: F. Gori, L. Geronazzo and M. Galeotti (eds.), Nonlinear Dynamics in Economics and the Social Sciences (Springer-Verlag, Berlin, 1993) pp. 69-94.

[3] R.H. Day and G. Pianigiani, Statistical dynamics and economics, Journal of Economic Behaviour and Organization 16 (1991) 37-83.

[4] T.M. Doup, A.H. van den Elzen, and A.J.J. Talman, Simplicial algorithms for solving the nonlinear complementarity problem on the simplotope, in A.J.J. Talman and G. van der Laan (eds.), The computation and modelling of economic equilibria NorthHolland, Amsterdam (1987) pp. 125-154.

[5] T.M. Doup and A.J.J. Talman, A new simplicial variable dimension algorithm to find equilibria on the product space of unit simplices, Mathematical Programming 37 (1987) 319-355.

[6] J.H. Drèze, Existence of an exchange economy under price rigidities, International Economic Review 16 (1975) 310-320.

[7] J.H. Drèze, Stability of a Keynesian adjustment process, in: W. Barnett, B. Cornet, C. d'Aspremont, J. J. Gabszewicz and A. Mas-Colell (eds.), Equilibrium Theory and Applications (Cambridge University Press, Cambridge, 1991).

[8] C.B. Garcia and W.I. Zangwill, Pathways to solutions, fixed points, and equilibria, Prentice-Hall Series in Computational Mathematics (Prentice-Hall, Englewood Cliffs, 1981). 
[9] P.J.J. Herings, A globally and universally stable price adjustment process, CentER Discussion Paper 9452, Tilburg University, Tilburg (1994), to appear in Journal of Mathematical Economics.

[10] P.J.J. Herings, A globally and universally stable quantity adjustment process for an economy with price rigidities, CentER Discussion Paper 94111, Tilburg University, Tilburg (1994).

[11] P.J.J. Herings, G. van der Laan, A.J.J. Talman, and R. Venniker, Equilibrium adjustment of disequilibrium prices, Discussion Paper TI 94-103, Tinbergen Institute, Amsterdam-Rotterdam (1994), to appear in Journal of Mathematical Economics.

[12] W. Hildenbrand, Core and equilibria of a large economy (Princeton University Press, Princeton, 1974).

[13] K. Kamiya, A globally stable price adjustment process, Econometrica 58 (1990) 14811485.

[14] H.J. Keisler, Approximate tâtonnement processes, Economic Theory 5 (1995) 127-173.

[15] H.J. Keisler, Getting to a competitive equilibrium, Econometrica 64 (1996) 29-49.

[16] G. van der Laan and A.J.J. Talman, Adjustment processes for finding economic equilibria, in: A.J.J. Talman and G. van der Laan (eds.), Computation and Modelling of Economic Equilibria (North-Holland, Amsterdam, 1987) pp. 85-123.

[17] G. van der Laan and A.J.J. Talman, A convergent price adjustment process, Economics Letters 23 (1987) 119-123.

[18] G. Laroque, A comment on "Stable spillovers among substitutes", Review of Economic Studies 48 (1981) 355-361.

[19] C.E. Lemke and J.T. Howson, Equilibrium points of bimatrix games, SIAM Journal of Applied Mathematics 12 (1964) 413-423.

[20] A. Mas-Colell, The theory of general economic equilibrium, a differentiable approach (Cambridge University Press, Cambridge, 1985).

[21] S.M. Movshovich, A price adjustment process in a rationed economy, Journal of Mathematical Economics 23 (1994) 305-321.

[22] A. Mukherji, A locally stable adjustment process, Econometrica 63 (1995) 441-448. 
[23] D.G. Saari and C. Simon, Effective price mechanisms, Econometrica 46 (1978) 1097 1125.

[24] H. Scarf, Some examples of global instability of the competitive equilibrium, International Economic Review 1 (1960) 157-172.

[25] H. Scarf, The Computation of Economic Equilibria (Yale University Press, New Haven, 1973).

[26] S. Smale, A convergent process of price adjustment and global Newton methods", Journal of Mathematical Economics 3 (1976) 107-120.

[27] M.J. Todd, The computation of fixed points and applications, Lecture notes in economics and mathematial systems 124 (Springer-Verlag, Berlin, 1976).

[28] E.C.H. Veendorp, Stable spillovers among substitutes, Review of Economic Studies 42 (1975) 445-456. 